\title{
Local Thermal Equilibrium States and Quantum Energy Inequalities
}

\author{
Jan Schlemmer and Rainer Verch
}

\begin{abstract}
In this paper we investigate the energy distribution of states of a linear scalar quantum field with arbitrary curvature coupling on a curved spacetime which fulfill some local thermality condition. We find that this condition implies a quantum energy inequality for these states, where the (lower) energy bounds depend only on the local temperature distribution and are local and covariant (the dependence of the bounds other than on temperature is on parameters defining the quantum field model, and on local quantities constructed from the spacetime metric). Moreover, we also establish the averaged null energy condition (ANEC) for such locally thermal states, under growth conditions on their local temperature and under conditions on the free parameters entering the definition of the renormalized stress-energy tensor. These results hold for a range of curvature couplings including the cases of conformally coupled and minimally coupled scalar field.
\end{abstract}

\section{Introduction}

One of the problems in quantum field theory is that the space of states is enormous, and that it is difficult to establish criteria which single out the states of interest in various physical situations. While this problem is met only in a mild form when considering quantum fields on Minkowski spacetime, it becomes quite pressing when trying to combine quantum field theory and gravity. A situation where this is predominant is quantum field theory in curved spacetime, where quantized matter fields propagate on a classical background spacetime which may be curved, and where the spacetime metric is not stationary - spacetimes of Friedman-RobertsonWalker type, for instance, are of particular interest in this context as they are simple models for cosmological scenarios. In trying to model the conditions of the early stages of the universe, one would like to distinguish quantum field states which are, at least locally, not too far from thermal equilibrium, and for which one can assign, at least locally, a temperature. It is not at all easy to arrive at a meaningful 
concept of temperature for states of a quantum field on a generic spacetime since the (global) notion of temperature makes reference to a global inertial frame, and that is not available in the presence of spacetime curvature. Another manifestation of this circumstance is the observer-dependence of the concepts of particle and temperature, as illustrated by the Fulling-Unruh, and related effects $[23,24,37]$.

Nevertheless, following a proposal by Buchholz, Ojima and Roos [9] and further investigated in $[8,10]$, it is possible to introduce a covariant concept of states which, at given points $x$ in spacetime, look like thermal equilibrium states with respect to a certain set, $S_{x}$, of reference-observables. Such states are called local thermal equilibrium (LTE) states with respect to $S_{x}$. While there is some leeway in the determination of $S_{x}$, it is important that $S_{x}$ does not contain observables which are sensitive to flux-like quantities, but instead observables which correspond, in the situation of global thermal equilibrium states defined with respect to an inertial frame (in the absence of spacetime curvature), to intensive thermal quantities. For a (scalar) quantum field $\phi(x)$, typical elements of $S_{x}$ are the Wick-square : $\phi^{2}:(x)$ of the field and its so-called "balanced derivatives". We will discuss the concept of LTE states further in the next section. However, it is important to mention that for an LTE state $\omega$ of the massless linear scalar field on a generic (globally hyperbolic) spacetime, the expectation value of the Wick-square of the field at any spacetime point $x$,

$$
\mathrm{T}^{2}(x)=\left\langle: \phi^{2}:(x)\right\rangle_{\omega} \equiv \omega\left(: \phi^{2}:(x)\right)
$$

equals, up to a constant, the square of the absolute temperature of the state at $x$. (A similar statement holds for the linear scalar field with positive mass parameter.)

Quite clearly, the local thermodynamic properties of states are linked with the local energetic properties of states, and investigation of that relation is the topic of the present article. A quantity of prominent interest in quantum field theory in curved spacetime - and with relevance to questions in cosmology - is the expectation value of the stress-energy tensor, $\left\langle T_{a b}(x)\right\rangle_{\omega}$, in a state $\omega$ for a quantum field on a curved spacetime. It becomes particularly important when considering the semiclassical Einstein equations (in geometric units),

$$
G_{a b}(x)=8 \pi\left(T_{a b}^{\text {class }}(x)+\left\langle T_{a b}(x)\right\rangle_{\omega}\right),
$$

where $G_{a b}(x)$ is the Einstein tensor of the spacetime geometry at spacetime point $x$ and $T_{a b}^{\text {class }}$ is the stress-energy tensor of macroscopically modelled (classical) matter. If the gravitational curvature effects caused by the macroscopic energy/matter distribution are very high, this may induce quantum field theoretical "particle creation effects", as a result of which $\left\langle T_{a b}(x)\right\rangle_{\omega}$ may turn out as a significant correction to the macroscopic matter distribution, depending on the quantum state $\omega$. One may expect that this is a realistic scenario as nowadays the Casimir force puts limits to the design of devices in micro- and nano-technology [4,35], and this effect can be seen in a similar vein.

One of the interesting features of the expectation value of stress energy is that the energy density seen by an observer travelling on a timelike geodesic $\gamma$ 
with tangent vector $v^{a}$ at $x,\left\langle T_{a b}(x)\right\rangle_{\omega} v^{a} v^{b}$, is unbounded above and below as $\omega$ ranges over the set of all states $\omega$ (for which the expectation value of stress-energy at any spacetime point $x$ can be reasonably defined). This is a long known feature of quantum field theory (see [11]) and is in contrast to the behaviour of macroscopic matter which can - with good motivation - usually be assumed to satisfy one of the classical energy conditions, like the weak energy condition, which means $T_{a b}^{\text {class }}(x) v^{a} v^{b} \geq 0$, i.e. the energy density seen by any observer is always positive at any spacetime point $x$.

Energy positivity conditions like the (pointwise) weak energy conditions play an important role in the derivation of singularity theorems $[25,40]$. One consequence of energy positivity conditions when plugged into Einstein's equations is that gravitational interaction is always attractive. Negative energies, in contrast, would be affected by a repelling gravitational interaction. This could, a priori, lead to solutions of Einstein's equations exhibiting very strange spacetime geometries, such as spacetimes with closed timelike curves, wormholes or "warpdrive scenarios" $[1,30]$. Moreover, concentration of a vast amount of negative energies and their persistence over a long duration could lead to violations of the second law of thermodynamics.

Motivated by the latter point, L. Ford has proposed that physical states of quantum fields in generic spacetimes should not permit arbitrary concentration of large amounts of negative energy over a long duration [21]. Such limitations on physical quantum field states have come to be called quantum energy inequalities $(Q E I s)$. Let us explain this concept in greater detail. Suppose that $\phi(x)$ is a quantum field on a generic spacetime. (Actually, only smeared quantum field quantities like $\phi(f)=\int f(x) \phi(x) d \operatorname{vol}(x)$ define proper quantum field operators operators; $d \operatorname{vol}(x)$ denotes the metric induced spacetime volume form and $f$ a smooth, compactly supported test-function. The quantum field can be of general spinor- or tensor type, but we suppress any corresponding indices here.) Then let $\mathcal{L}$ be a set of states of the quantum field such that the expectation value of the stress-energy tensor, $\left\langle T_{a b}(x)\right\rangle_{\omega}$, is defined for each $\omega \in \mathcal{L}$ at each spacetime point $x$. We will furthermore suppose that this quantity is continuous in $x$ for each $\omega \in \mathcal{L}$. Under these assumptions, we say that the set of quantum field states $\mathcal{L}$ fulfills a QEI with respect to $\gamma$ if

$$
\int h^{2}(t)\left\langle T_{a b}(\gamma(t))\right\rangle_{\omega} \dot{\gamma}^{a}(t) \dot{\gamma}^{b}(t) d t \geq q(\gamma, h)
$$

holds for all smooth (or at least $C^{2}$ ) real functions $h$ having compact support on the (open) curve domain, with a constant $q(\gamma, h)>-\infty$; the constant may depend on the curve $\gamma$ and the weighting function $h$, but is required to be independent of the choice of state $\omega \in \mathcal{L}$.

In principle this concept makes sense for arbitrary ( $C^{1}$ and causal) curves $\gamma$, in practice one however usually restricts the class of curves to timelike (or lightlike) geodesics. Such a limiting case of a QEI is the following: If $\gamma$ is a complete (lightlike or null) geodesic, then one says that a set of states $\mathcal{L}$ fulfills the averaged null energy 
condition (ANEC) if

$$
\liminf _{\lambda \rightarrow 0+} \int h^{2}(\lambda t) \dot{\gamma}^{a}(t) \dot{\gamma}^{b}(t)\left\langle T_{a b}(\gamma(t))\right\rangle_{\omega} d t \geq 0
$$

holds for all states $\omega \in \mathcal{L}$. Conditions of such form (and related conditions, see (39)), if valid for all complete null geodesics, allow conclusions about focussing of null geodesics for solutions to the semiclassical Einstein equations similar to that resulting from a pointwise null energy condition $[5,32,36,42]$. (See also the beginning of Section 4.) Thus, the ANEC is a key property for deriving singularity theorems for solutions to the semiclassical Einstein equations.

Quantum energy inequalities have been investigated extensively for quantum fields subject to linear field equations in the recent years, and there is now a wealth of results in this regard. We refer to the reviews by Fewster and by Roman $[13,33]$ for representative lists of references. Important to mention, however, is the fact that for many linear fields, like the minimally coupled scalar field, the Dirac field and the electromagnetic field, it could be shown that the set of Hadamard states fulfills a QEI with respect to timelike curves $\gamma$ in generic globally hyperbolic spacetimes $[12,15,19]$. Hadamard states are regarded as physical states in quantum field theory in curved spacetime, and expectation values of the stress-energy tensor at any given spacetime point are well-defined for these states (up to finite renormalization ambiguities), cf. [41] for discussion. There is also an intimate relation between QEIs, the Hadamard condition and thermodynamic properties of linear quantum fields [20]. It has been shown that QEIs put strong limitations on the possibility of solutions to the semiclassical Einstein's equations to allow exotic spacetime scenarios such as wormholes or warpdrive $[17,22,31]$. It is also worth mentioning two other recent results. First, it has been shown that the non-minimally coupled linear scalar field on any spacetime violates QEIs for the class of Hadamard states; nevertheless, the class of Hadamard states fulfills in this case weaker bounds, called "relative QEIs", cf. [14] for results and discussion. Secondly, one is interested in lower bounds $q(\gamma, h)$ which depend (apart from renormalization constants entering the definition of expectation value of the stress energy tensor) only on the underlying spacetime geometry in a local and covariant manner, and one also aims at making this dependence as explicit as possible. Considerable progress on this issue, for the case of the minimally coupled linear scalar field on globally hyperbolic spacetimes, has been achieved in [18].

In the present article, we will derive QEI-like bounds on sets of LTE-states of the non-minimally coupled linear scalar field $\phi(x)$ on generic globally hyperbolic spacetimes. More precisely, we consider LTE states $\omega$ whose thermal function $\vartheta^{\omega}(x)=\left\langle: \phi^{2}:(x)\right\rangle_{\omega}$ is bounded by some constant $\mathrm{T}_{0}^{2}$ (corresponding to a maximal squared temperature) and we will show that there are upper and lower bounds for the averaged energy density

$$
\int_{-\infty}^{\infty} \eta(\tau) v^{a} v^{b}\left\langle T_{a b}(\gamma(\tau))\right\rangle_{\omega} d \tau
$$


averaged against a $C^{2}$-weighting function $\eta \geq 0$ with compact support along any causal geodesic $\gamma$ with affine parameter $\tau$ and tangent $v^{a}=\dot{\gamma}^{a}$. The lower bound depends only on $\mathrm{T}_{0}^{2}$, the geodesic $\gamma$ and $\eta$, while the upper bound depends additionally on local tetrads entering into the definition of LTE states. The lower bound is therefore state-independent within each set of LTE states $\omega$ with a fixed maximal value of $\vartheta^{\omega}$. The bounds depend on the spacetime geometry in a local covariant manner which, together with their dependence on $\mathrm{T}_{0}^{2}$, we will make explicit. This result holds for all values of curvature coupling $\xi$ in the field equation (4), and upon averaging along causal geodesic, not only those which are timelike. Hence, the result is not immediate from know quantum energy inequalities for Hadamard states, as these are violated in general for non-minimally coupled fields [14], and upon averaging along null geodesics [16]. Furthermore, we will show that the ANEC holds for LTE states $\omega$ of the quantized linear scalar field with curvature couplings $0 \leq \xi \leq 1 / 4$, provided that the growth of the thermal function $\vartheta^{\omega}$ along the null geodesics $\gamma$ fulfills certain bounds. Despite the fact that we have to assume that the LTE states we consider are Hadamard states - in order to have a well-defined, local covariant expression of expected stress-energy for these states - our derivation of QEIs and ANEC makes no further use of the Hadamard property but uses only properties of LTE states. Therefore, one may expect that, in principle, similar results could be derived for LTE states of interacting quantum fields. This prospect can actually be seen as one of our motivations in view of the fact that quantum energy inequalities seem to be very difficult to obtain (if valid at all) for very general sets of states in interacting quantum field theory, and that, on the other hand, one may argue that only special classes of states are of physical interest. We will come back to this point in Section 6 .

This article is organized as follows. We will discuss the concept of LTE states, as far as needed for our purposes, in Section 2. In Section 3 we derive upper and lower bounds for the geodesically averaged expectation values of energy density for LTE states. The validity of ANEC for certain LTE states will be studied in Section 4. In Section 5 we indicate that the results of Sections 3 and 4 hold also for a more general notion of LTE states. We conclude with discussion and outlook in Section 6.

\section{Local thermal equilibrium states}

The system under investigation in the present article is the non-minimally coupled linear scalar field on globally hyperbolic spacetimes. A globally hyperbolic spacetime will be denoted by a pair $(M, g)$ where $M$ is the spacetime manifold (assumed to be $C^{\infty}$ ) and $g$ is the Lorentzian metric. We will consider the case of spacetime dimension equal to 4 with metric signature $(+---)$, but most of our considerations can be readily generalized, with appropriate modifications, to arbitrary spacetime dimensions. We recall that global hyperbolicity means that 
the spacetime is time-orientable and possesses Cauchy surfaces $[2,40]$. Our conventions for curvature quantities, like in [14], are those of Birrell and Davies, i.e. $[-,-,-]$ in the classification scheme of Misner, Thorne and Wheeler.

The classical linear scalar field $\varphi$ on a globally hyperbolic spacetime $(M, g)$ obeys the field equation

$$
\left(\nabla^{\mu} \nabla_{\mu}+\xi R+m^{2}\right) \varphi=0
$$

where $\nabla$ is the covariant derivative of $g$ and $R$ is the scalar curvature corresponding to $g$; the constants $\xi \geq 0$ and $m \geq 0$ are the curvature coupling and the mass parameters, respectively. The case $\xi=0$ corresponds to minimal coupling.

The quantization of the system proceeds as follows. Owing to global hyperbolicity, there are (for each fixed $\xi$ and $m$ ) two uniquely determined linear maps $E^{ \pm}: C_{0}^{\infty}(M, \mathbb{R}) \rightarrow C^{\infty}(M, \mathbb{R})$ so that

$$
E^{ \pm}\left(\nabla^{\mu} \nabla_{\mu}+\xi R+m^{2}\right) f=f=\left(\nabla^{\mu} \nabla_{\mu}+\xi R+m^{2}\right) E^{ \pm} f
$$

holds for all $f \in C_{0}^{\infty}(M, \mathbb{R})$, and additionally, $\operatorname{supp}\left(E^{ \pm} f\right) \subset J^{ \pm}(\operatorname{supp}(f))$, where $J^{ \pm}(G)$ is the causal future/past set of $G \subset M[2]$. These are called the advanced/retarded fundamental solutions of the wave-operator $\left(\nabla^{\mu} \nabla_{\mu}+\xi R+m^{2}\right)$, and with their help one can construct the real bilinear form

$$
\mathscr{E}\left(f_{1}, f_{2}\right)=\int_{M} f_{1}(x)\left(E^{-} f_{2}-E^{+} f_{2}\right)(x) d \operatorname{vol}(x)
$$

on $C_{0}^{\infty}(M, \mathbb{R})$ which turns out to be antisymmetric. Note that $\mathscr{E}$ is uniquely determined by $(M, g), \xi$ and $m$. Fixing $\xi$ and $m$, one can now define the complex *-algebra $\mathcal{A}(M, g)=\mathcal{A}((M, g), \xi, m)$ with unit element 1 as being generated by a family of objects $\phi(f), f \in C_{0}^{\infty}(M, \mathbb{R})$ which are required to fulfil the following relations:

(a) $f \mapsto \phi(f)$ is real-linear,

(b) $\phi(f)^{*}=\phi(f)$,

(c) $\phi\left(\left(\nabla^{\mu} \nabla_{\mu}+\xi R+m^{2}\right) f\right)=0$,

(d) $\left[\phi\left(f_{1}\right), \phi\left(f_{2}\right)\right]=i \mathscr{E}\left(f_{1}, f_{2}\right) 1$.

Here $[A, B]=A B-B A$ denotes the commutator. Since the generators $\phi(f)$ of $\mathcal{A}(M, g)$ obey, according to (d), the canonical commutation relations in a covariant manner, one has thus obtained a quantization of the system in an abstract form. The hermitean elements in $\mathcal{A}(M, g)$ correspond to observables of the quantized system, but they do not contain all observables that one may wish to consider, so that the algebra $\mathcal{A}(M, g)$ will have to be enlarged to include those additional observables as well. We will come back to this point. For the moment, a state $\omega$ of the quantized linear scalar field is, by definition, a linear functional $\omega: \mathcal{A}(M, g) \rightarrow$ $\mathbb{C}, A \mapsto \omega(A) \equiv\langle A\rangle_{\omega}$, with the additional property that $\omega$ is positive, meaning $\omega\left(A^{*} A\right) \geq 0$ for all $A \in \mathcal{A}(M, g)$, and also with the property that $\omega$ is normalized, i.e. $\omega(1)=1$. Now it is known from examples that not every state according to this definition corresponds to a physically reasonable configuration of the system and that selection criteria for physical states are needed. In the case of the linear 
fields on curved spacetime, the best candidates for physical states are quasifree Hadamard states, and most other physical states can be derived from those [38].

We will very briefly summarize the concept of a quasifree Hadamard state. (For a more in-depth discussion, see [28].) For any state $\omega$ on $\mathcal{A}$, the $n$-point functions are the maps

$$
f_{1} \otimes \cdots \otimes f_{n} \mapsto \mathscr{W}_{n}^{\omega}\left(f_{1}, \ldots, f_{n}\right)=\omega\left(\phi\left(f_{1}\right) \cdots \phi\left(f_{n}\right)\right) .
$$

Clearly, each state on $\mathcal{A}(M, g)$ is determined by all the $n$-point functions. A quasifree state $\omega$ on $\mathcal{A}(M, g)$ is a state which is entirely determined by its twopoint function, by requiring that the truncated $n$-point functions vanish [6]. This can also be expressed as $\omega\left(\mathrm{e}^{i t \phi(f)}\right)=\mathrm{e}^{-t^{2} \mathscr{W}_{2}^{\omega}(f, f) / 2}$, to be interpreted as a sequence of relations in the sense of formal power series in $t$. A (quasifree) state $\omega$ is called Hadamard state if its two-point function is of Hadamard form. This is the case, in turn, if for any geodesic convex neighbourhood $N$ of any given point $x_{o}$, and any time function $t$ on the underlying spacetime $M$, one can find a sequence $H_{k}^{\omega} \in C^{k}(N \times N, \mathbb{C})$ such that for all $f_{1}, f_{2} \in C_{0}^{\infty}(N, \mathbb{R})$ one has

$$
\begin{aligned}
& \mathscr{W}_{2}^{\omega}\left(f_{1}, f_{2}\right) \\
& =\lim _{\varepsilon \rightarrow 0+} \frac{1}{4 \pi^{2}} \int_{N \times N}\left(G_{k, \varepsilon}\left(x, x^{\prime}\right)+H_{k}\left(x, x^{\prime}\right)\right) f_{1}(x) f_{2}\left(x^{\prime}\right) d \operatorname{vol}(x) d \operatorname{vol}\left(x^{\prime}\right),
\end{aligned}
$$

where

$$
\begin{aligned}
G_{k, \varepsilon}\left(x, x^{\prime}\right)= & \frac{U\left(x, x^{\prime}\right)}{\sigma\left(x, x^{\prime}\right)+2 i\left(t(x)-t\left(x^{\prime}\right)\right) \varepsilon+\varepsilon^{2}} \\
& +\mathcal{V}_{k}\left(x, x^{\prime}\right) \ln \left(\sigma\left(x, x^{\prime}\right)+2 i\left(t(x)-t\left(x^{\prime}\right)\right) \varepsilon+\varepsilon^{2}\right) .
\end{aligned}
$$

Here,

$$
\mathcal{V}_{k}\left(x, x^{\prime}\right)=\sum_{j=0}^{k} U_{j}\left(x, x^{\prime}\right) \sigma\left(x, x^{\prime}\right)^{j},
$$

$\sigma\left(x, x^{\prime}\right)$ is the squared geodesic distance from $x$ to $x^{\prime 1}$, and $U$ and $U_{j}$ are smooth functions on $N \times N$ determined by the Hadamard recursion relations. Thus, the term $G_{k, \varepsilon}$ is, for each $k$, determined by the local spacetime geometry and the parameters $\xi$ and $m$ of the scalar field equation. For later use, we define the distribution

$$
\mathscr{G}_{k}\left(f_{1}, f_{2}\right)=\lim _{\varepsilon \rightarrow 0+} \frac{1}{4 \pi^{2}} \int G_{k, \varepsilon}\left(x, x^{\prime}\right) f_{1}(x) f_{2}\left(x^{\prime}\right) d \operatorname{vol}(x) d \operatorname{vol}\left(x^{\prime}\right)
$$

for test-functions $f_{1}, f_{2}$ supported in a geodesic convex neighbourhood $N$.

It is worth noting that the existence of very many quasifree Hadamard states (spanning an infinite dimensional space) has been established for the linear scalar

${ }^{1}$ Following [18], we choose $\sigma$ positive for $x$ and $x^{\prime}$ spacelike related and negative for $x$ and $x^{\prime}$ timelike related, so that e.g. on Minkowski spacetime we have $\sigma\left(x, x^{\prime}\right)=-g_{a b}\left(x-x^{\prime}\right)^{a}\left(x-x^{\prime}\right)^{b}$ 
field on all globally hyperbolic spacetimes. Moreover, in stationary, globally hyperbolic spacetimes, the canonical ground state as well as the thermal equilibrium states (KMS states) are known to be quasifree Hadamard states [34].

Let us now turn to the concept of local thermal equilibrium states introduced in [9] and further investigated in $[8,10]$. This will be done first for the case that the underlying spacetime is just Minkowski spacetime. Our discussion here is limited to the linear scalar field, but as explained in [9], the discussion can be generalized to include general quantum field theories. For the quantized linear scalar field on Minkowski spacetime, there is in each Lorentz frame a unique quasifree thermal equilibrium state at given temperature. Actually, for fixed temperature this state depends only on the time-direction of the Lorentz frame. Let $e_{0}$ be such a time-direction, i.e. a timelike, future-pointing unit vector on Minkowski spacetime, and let $e_{1}, e_{2}, e_{3}$ be a set of spacelike unit vectors so that $e=\left(e_{0}, e_{1}, e_{2}, e_{3}\right)$ forms an orthonormal tetrad on Minkowski spacetime. When choosing coordinates $\left(x^{0}, x^{1}, x^{2}, x^{3}\right)$ on Minkowski spacetime such that the coordinate axes are aligned with the tetrad, the two-point function $\mathscr{W}_{2}^{\beta e}$ of the unique quasifree thermal equilibrium (KMS) state $\omega^{\beta e}$ at inverse temperature $\beta>0{ }^{2}$ with respect to the Lorentz frame defined by $e$ is given by

$$
\mathscr{W}_{2}^{\beta e}\left(x, x^{\prime}\right)=\int \mathrm{e}^{-i\left(x-x^{\prime}\right)^{\mu} p_{\mu}} \epsilon\left(p_{0}\right) \delta\left(p_{\mu} p^{\mu}-m^{2}\right) \frac{d^{4} p}{(2 \pi)^{3}\left(1-\mathrm{e}^{-\beta p_{0}}\right)},
$$

to be interpreted in the sense of distributions, where $\epsilon\left(p_{0}\right)$ is the sign function of $p_{0}$. The uniqueness implies that every intensive thermal property (e.g. pressure, density etc) can be expressed as a function of the timelike vector $\beta e_{0}$.

The passage from these global equilibrium states to states of local thermodynamic equilibrium now uses spaces $S_{x}$ of observables located at spacetime points $x$. Mathematically this implies that these observables are no longer defined as operators but only as quadratic forms (their products are usually not defined). In physical terms, the observables in $S_{x}$ should model idealized limits of measurements of intensive thermal properties of states in smaller and smaller spacetime regions. This culminates in the requirement that, for $s(x) \in S_{x}$, the functions

$$
\Phi_{s}(x)=\omega^{\beta e}(s(x))
$$

have to be independent of $x$ and non-constant as functions of $\beta$. Furthermore, for many $s(x) \in S_{x}$ one can identify the thermal quantity to which $\Phi_{s}$, as a function of $\beta$, actually corresponds. As an example, one calculates that for $s(x)=: \phi^{2}:(x)$ (Wick-square) one obtains for the massless case

$$
\Phi_{: \phi^{2}:}=\omega^{\beta e}\left(: \phi^{2}:(x)\right)=\frac{1}{12 \beta^{2}}=\frac{k_{B}^{2} \mathrm{~T}^{2}}{12}
$$

which leads to the identification of this observable as a "scalar thermometer", giving the square of the local temperature $\mathrm{T}$ times some fixed constant. Choosing appropriate units to measure $\mathrm{T}$, this constant can be set equal to 1 , justifying

${ }^{2} \beta=1 /\left(k_{B} \mathrm{~T}\right)$ where $k_{B}$ is Boltzmann's constant and $\mathrm{T}$ is absolute temperature 
the notation $\mathrm{T}^{2}$ for the expectation value of the Wick-square in thermal equilibrium states already alluded to above. Actually this seems quite similar to what one would do to construct a thermometer in the laboratory: Take some (small) device which, when exposed to a situation known to be in equilibrium at some temperature $\mathrm{T}$, gives a reading which is a simple function of $\mathrm{T}$.

In the investigations of local thermal equilibrium for linear scalar fields $\phi(x)$ on Minkowski spacetime carried out in previous articles [8-10], the spaces $S_{x}$ are chosen as the linear spaces generated by elements $s(x)=\widetilde{\partial}_{\mu}: \phi^{2}:(x)$, referred to as the balanced derivatives of the Wick-squared field $: \phi^{2}:(x) .{ }^{3}$ Here, $\boldsymbol{\mu}=\left(\mu_{1}, \ldots, \mu_{n}\right) \in \mathbb{N}^{n}$ is a multi-index of arbitrary length $n$, and the balanced derivatives are defined by

$$
\check{\partial}_{\boldsymbol{\mu}}: \phi^{2}:(x)=\lim _{\zeta \rightarrow 0} \partial_{\boldsymbol{\mu}}\left(\phi(x+\zeta) \phi(x-\zeta)-\omega^{\mathrm{vac}}(\phi(x+\zeta) \phi(x-\zeta)) 1\right)
$$

where $\partial_{\boldsymbol{\mu}}=\partial_{\zeta^{\mu_{1}}} \cdots \partial_{\zeta^{\mu_{n}}}$, and where $\omega^{\text {vac }}$ is the vacuum state. The limit is taken along spacelike directions $\zeta$, so that $\phi(x+\zeta) \phi(x-\zeta)$ is well defined as a quadratic form, and the limit defines an operator-valued distribution after smearing in $x$ with test-functions. For multi-index length equal to 0 , the balanced derivative equals just the Wick-square : $\phi^{2}:(x)$. For linear fields on Minkowski spacetime, this definition of the Wick-square coincides with the usual normal ordering prescription. Owing to the translation invariance of the KMS-states $\omega^{\beta e}$ one can easily check that the thermal functions $\Phi_{\varlimsup_{\mu}: \phi^{2}:}=\omega^{\beta e}\left(ð_{\mu}: \phi^{2}:(x)\right)$ are independent of $x$.

Following [8,9], a state of the linear scalar field on Minkowski spacetime is said to be locally in thermal equilibrium at a spacetime point $x$ if it looks like a global thermal equilibrium state $\omega^{\beta e}$ as far as the expectation values of elements in $S_{x}$ are concerned. The following definition, taken from [9], expresses this more formally.

Definition 2.1. A state $\omega$ of the quantized linear scalar field $\phi(x)$ on Minkowski spacetime is called $S_{x}$-thermal at the spacetime point $x$ if there are an orthonormal tetrad $e$ with $e_{0}$ timelike and future-pointing, and $\beta>0$, such that

$$
\omega(s(x))=\omega^{\beta e}(s(x))
$$

holds for all $s(x) \in S_{x}$, where $S_{x}$ is spanned by $\partial_{\boldsymbol{\mu}}: \phi^{2}:(x)$ as $\boldsymbol{\mu}$ ranges over all multi-indices.

If $\mathcal{O}$ is some open set of spacetime points, a state $\omega$ of the quantized linear scalar field on Minkowski spacetime is called $S_{\mathcal{O}}$-thermal if (14) holds and $\omega(s(x))$ varies continuously with $x$, for $s(x) \in S_{x}$ and $x \in \mathcal{O}$. That means, $\omega$ is $S_{x}$-thermal at each $x \in \mathcal{O}$, where $\beta$ and $e$ in (14) may vary with $x$.

For $S_{\mathcal{O}}$-thermal states, the expectation value $\left\langle\Phi_{s}\right\rangle_{\omega}(x)$ of an extensive thermal quantity $\Phi_{s}$ at $x$, whose local measurement is modelled by $s(x) \in S_{x}$, is then given as $\left\langle\Phi_{s}\right\rangle_{\omega}(x)=\omega(s(x))$. This leads for $S_{\mathcal{O}}$-thermal states to an assignment of thermal quantities to each $x \in \mathcal{O}$ whose values in general vary with $x$, and this

\footnotetext{
${ }^{3}$ In the references $[8,9]$, the notation $\check{\partial}^{\mu}$ is used, but we prefer to view $\boldsymbol{\mu}$ as a co-tensor index
} 
assignment is consistent in the sense that relations among the thermal quantities (like equations of state) also hold at each point. In the case of $s(x)=: \phi^{2}:(x)$, one obtains in this way for an $S_{\mathcal{O}}$-thermal state $\omega$ an assignment of the expected squared temperature to each spacetime point $x \in O$.

The concept of states which are locally in thermal equilibrium has also been generalized in [9] to allow mixtures of global thermal equilibrium states on the right hand side of (14). We will summarize this generalized concept in Section 5.

In attempting to extend the concept of local thermal equilibrium states to quantum fields in curved spacetime, one faces a couple of difficulties which are, of course, connected to the occurrence of curvature and the related lack of global vacuum states and global equilibrium states. Primarily, these difficulties are:

(i) The definition (13) of balanced derivatives $\partial_{\mu}: \phi^{2}:(x)$ uses the Minkowski vacuum state $\omega^{\mathrm{vac}}$ as preferred vacuum state.

(ii) Moreover, the definition (13) uses the affine space structure of Minkowski spacetime.

(iii) Definition 2.1 uses global thermal equilibrium states $\omega^{\beta e}$ on Minkowski spacetime, for which there is no counterpart on generic curved spacetimes.

Thus, there is no verbatim translation of the concept of local thermal equilibrium states given in Definition 2.1.

It is clear that problems (i) and (ii) concern the definition of balanced derivatives of a Wick-squared quantum field in curved spacetime. We will soon turn to that problem. Assuming that the definition of balanced derivatives in curved spacetime is settled, a proposal was made in [10] to surpass problem (iii). The idea is to define that a state $\omega$ of the quantized linear scalar field $\phi$ on a curved spacetime $(M, g)$ is $S_{x}$-thermal at a point $x$ in $M$ if $\omega(s(x))=\omega_{o}^{\beta e}\left(s_{o}\left(x_{o}\right)\right)$ holds for all $s(x) \in S_{x}$. Here, $\omega_{o}^{\beta e}$ is a thermal equilibrium state of the free scalar field $\phi_{o}$ (with same parameters as $\phi$ ) on Minkowski spacetime $M_{o}, x_{o}$ is a point in $M_{o}$, and $s_{o}\left(x_{o}\right)$ is the flat space counterpart of $s(x)$. To explain what this latter phrase means precisely can be seen as part of the definition of balanced derivatives in curved spacetime, but certainly one would require that $s_{o}\left(x_{o}\right)$ corresponds to a balanced derivative of the Wick-square of $\phi_{o}$ if $s(x)$ corresponds to a balanced derivative of the Wick-square of $\phi$. In the approach of [10], the requirement of local thermality on $\omega$ is thus not implemented by comparing expectations values of pointlike thermal observables with the corresponding expectation values in a global thermal state (as such states need not exist), but with the "flat space version" of a thermal equilibrium situation for the quantum field. The motivation for this approach is that $S_{x}$-thermality is a pointwise property which should not be affected by curvature; this, in turn, rests largely on the equivalence principle.

For a linear scalar quantum field $\phi$ on a curved spacetime it is simple enough to know what its flat space counterpart $\phi_{o}$ should be. However, as pointed out in [10], one may invoke the concept of a local covariant quantum field theory $[7,26]$ to know this also for more general types of quantum fields. The concept of local covariance affects also the elements $s(x) \in S_{x}$; in our situation where we start 
from a linear scalar field $\phi$ in curved spacetime - which is known to have the structure of a local covariant quantum field - the balanced derivatives of Wicksquares of $\phi$ should be defined in such a way that they are also local covariant quantum fields.

Let us thus discuss our proposal for the generalization of the concept of balanced derivatives in a curved spacetime. Our discussion is greatly facilitated by the circumstance that for the purpose of deriving QEIs for LTE states we need only focus on balanced derivatives up to second order, corresponding to a multi-index length of $\boldsymbol{\mu}$ not greater than two. Accordingly, we will define the LTE property on curved spacetime only with balanced derivatives of the Wick-squared field up to second order, see below.

We proceed in two steps. First, we shall consider the generalization of expressions like $\left.\partial_{\zeta^{\mu}} \partial_{\zeta^{\nu}} f(x+\zeta, x-\zeta)\right|_{\zeta=0}$ for $C^{2}$-functions $f$ from Minkowski spacetime to curved spacetime. This discussion is entirely of differential geometric nature. In a second step, we have to give a generalization of the quantity

$$
f(x+\zeta, x-\zeta)=\omega(\phi(x+\zeta) \phi(x-\zeta))-\omega^{\operatorname{vac}}(\phi(x+\zeta) \phi(x-\zeta))
$$

for Hadamard states $\omega$ on curved spacetime where there is no counterpart of $\omega^{\text {vac }}$. In doing this we have to ensure, as mentioned, that the resulting balanced derivatives of the Wick-ordered linear scalar field give rise to local covariant quantum fields.

Turning to the first step, let $(M, g)$ be a spacetime and suppose that $N$ is a geodesically convex neighbourhood of some point $x$ in $M$. The exponential map at $x$ will be denoted by $\exp _{x}$. A fairly obvious generalization of the first balanced derivative of a function $f \in C^{2}(N \times N)$ arises by requiring

$$
v^{a} \check{\partial}_{a} f(x)=\left.\frac{d}{d \lambda}\right|_{\lambda=0} f\left(\exp _{x}(\lambda v), \exp _{x}(-\lambda v)\right)
$$

for all spacelike vectors $v=v^{a} \in T_{x} M$ lying in $\exp _{x}^{-1}(N)$. By linearity, this determines a co-vector $\partial_{a} f(x)$ in $T_{x}^{*} M$. We define $T\left(\begin{array}{cc}r & r^{\prime} \\ s & s^{\prime}\end{array}\right)(M \times M)$ as the bundle over $M \times M$ whose fibre at $\left(y, y^{\prime}\right) \in M \times M$ is given by $T_{y}\left(\begin{array}{l}r \\ s\end{array}\right) M \otimes T_{y^{\prime}}\left(\begin{array}{l}r^{\prime} \\ s^{\prime}\end{array}\right) M$, where $T_{y}\left(\begin{array}{l}r \\ s\end{array}\right) M$ coincides with the space of $r$-fold contravariant and $s$-fold covariant tensors

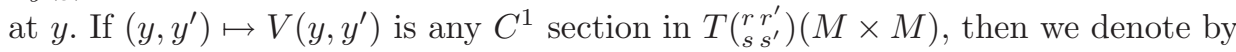
$\nabla_{a} V$ the covariant derivative with respect to the $y$-entry and by $\nabla_{a^{\prime}} V$ the covariant derivative with respect to the $y^{\prime}$-entry. Furthermore, we denote by $V\left\lfloor_{x}=V(x, x)\right.$ the coincidence value of $V\left(y, y^{\prime}\right)$ for $y=x=y^{\prime}$. With these conventions, one has

$$
\partial_{a} f(x)=\nabla_{a} f\left\lfloor_{x}-\nabla_{a^{\prime}} f\left\lfloor_{x},\right.\right.
$$

and if $f$ is $C^{2}$, this defines a $C^{1}$ co-vector field as $x$ varies.

The second order balanced derivative $\partial_{a b} f(x)$ can then be defined as follows. $\nabla_{a} f\left(y, y^{\prime}\right)$ is a $y^{\prime}$-dependent co-vector at $y$, and vice versa for $\nabla_{a^{\prime}} f\left(y, y^{\prime}\right)$. Let $v=v^{a}$ be a (spacelike) vector in $T_{x} M$ which lies in $\exp _{x}^{-1}(N)$, so that $\eta_{v}: \lambda \mapsto \exp _{x}(\lambda v)(-1 \leq \lambda \leq 1)$ is the geodesic determined by $v$ at $x$. Correspondingly, we can define the map of parallel transport $P_{v, \lambda}: T_{\exp _{x}(\lambda v)}^{*} M \rightarrow T_{x}^{*} M$ 
of co-vectors from $\exp _{\lambda v}=\eta_{v}(\lambda)$ to $x=\eta_{v}(0)$ along the geodesic $\eta_{v}$. A geometrically natural definition of the second order balanced derivative $\partial_{a b} f(x)$ of $f$ at $x$ is then obtained by demanding that

$$
\begin{aligned}
v^{a} w^{b \check{\partial}_{a b} f(x)=} & \left.\frac{d}{d \lambda}\right|_{\lambda=0} w^{b} P_{v, \lambda} \nabla_{b} f\left(\exp _{x}(\lambda v), \exp _{x}(-\lambda v)\right) \\
& -\left.\frac{d}{d \lambda}\right|_{\lambda=0} w^{b^{\prime}} P_{-v, \lambda} \nabla_{b^{\prime}} f\left(\exp _{x}(\lambda v), \exp _{x}(-\lambda v)\right)
\end{aligned}
$$

holds for all (spacelike) vectors $v, w \in T_{x} M$ with $v \in \exp _{x}^{-1}(N)$. Using the properties of the parallel transport, it follows that $M \ni x \mapsto \widetilde{\partial}_{a b} f(x)$ is a continuous (if $f$ is $C^{2}$ ) $\left(\begin{array}{l}0 \\ 2\end{array}\right)$-tensor field on $M$, and

$$
\begin{aligned}
\check{\partial}_{a b} f(x)= & \nabla_{a} \nabla_{b} f\left\lfloor_{x}-\nabla_{a} \nabla_{b^{\prime}} f\left\lfloor_{x}\right.\right. \\
& -\nabla_{a^{\prime}} \nabla_{b} f\left\lfloor_{x}+\nabla_{a^{\prime}} \nabla_{b^{\prime}} f\left\lfloor_{x} .\right.\right.
\end{aligned}
$$

Note here that the covariant derivatives on the right hand side act on $y$ for unprimed indices and on $y^{\prime}$ for primed indices, and primed and unprimed tensor indices are identified at the coincidence point $y=x=x^{\prime}$.

Looking at the final result obtained in (15) one notices that this is exactly what one would have obtained by replacing ordinary by covariant derivatives in the prescription for Minkowski spacetime. This is an indication that at the level of the second order balanced derivatives ordering problems for multiple (covariant) derivatives do not yet arise (as is to be expected for a torsion-free connection). Such ambiguities can however be expected when defining higher order balanced derivatives. Other possible modifications of the balanced derivatives include the addition of suitable geometric terms like $g_{a b} R$, but such terms will be dealt with in the transition from the classical to the quantum expressions anyway and it is the assumption of general covariance for the resulting quantum fields which then imposes restrictions on the possibility of such terms.

Turning to the second step, suppose that $(M, g)$ is a globally hyperbolic spacetime, and that $N$ is a geodesic convex neighbourhood of some point $x \in M$. Then define the distributions $(k \geq 2)$

$$
\tilde{\mathscr{G}}_{k}\left(f_{1}, f_{2}\right)=\frac{1}{2}\left(\mathscr{G}_{k}\left(f_{1}, f_{2}\right)+\mathscr{G}_{k}\left(f_{2}, f_{1}\right)\right)+\frac{i}{2} \mathscr{E}\left(f_{1}, f_{2}\right), \quad f_{1}, f_{2} \in C_{0}^{\infty}(N, \mathbb{R}),
$$

where $\mathscr{G}_{k}$ and $\mathscr{E}$ are defined above for the quantized linear scalar field $\phi$ on $(M, g)$.

Next, let $\omega$ be a quasifree Hadamard state of $\phi$ on $(M, g)$; then define the point-split renormalized two-point function obtained by subtracting the symmetrized Hadamard parametrix (SHP) $\widetilde{\mathscr{G}}_{k}$ from the two-point function:

$$
\mathscr{W}_{\omega, k}^{\mathrm{SHP}}\left(f_{1}, f_{2}\right)=\mathscr{W}_{2}^{\omega}\left(f_{1}, f_{2}\right)-\widetilde{\mathscr{G}}_{k}\left(f_{1}, f_{2}\right), \quad f_{1}, f_{2} \in C_{0}^{\infty}(N, \mathbb{R})
$$


A first observation is that $\mathscr{W}_{\omega, k}^{\mathrm{SHP}}$ is symmetric, $\mathscr{W}_{\omega, k}^{\mathrm{SHP}}\left(f_{1}, f_{2}\right)=\mathscr{W}_{\omega, k}^{\mathrm{SHP}}\left(f_{2}, f_{2}\right)$. Furthermore, for $k \geq 2, \mathscr{W}_{\omega, k}^{\mathrm{SHP}}$ is given

$$
\begin{aligned}
\mathscr{W}_{\omega, k}^{\mathrm{SHP}} & \left(f_{1}, f_{2}\right) \\
& =\int_{N \times N} W_{\omega, k}^{\mathrm{SHP}}\left(y, y^{\prime}\right) f_{1}(y) f_{2}\left(y^{\prime}\right) d \operatorname{vol}(y) d \operatorname{vol}\left(y^{\prime}\right), \quad f_{1}, f_{2} \in C_{0}^{\infty}(N, \mathbb{R}),
\end{aligned}
$$

with $W_{\omega, k}^{\text {SHP }} \in C^{2}(N \times N)$ for $k \geq 2$, which follows from the definition of Hadamard form (see also the arguments in [18]).

Consequently, one may now define for any quasifree Hadamard state $\omega$ the expectation value of the SHP-Wick square of $\phi$ and the corresponding second balanced derivatives in the following way:

\section{Definition 2.2.}

$$
\begin{aligned}
\omega\left(: \phi^{2}:_{\mathrm{SHP}}(x)\right) & =W_{\omega, k}^{\mathrm{SHP}}\left\lfloor_{x},\right. \\
\omega\left(\check{\partial}_{a b}: \phi^{2}:_{\mathrm{SHP}}(x)\right) & =\widetilde{\partial}_{a b} W_{\omega, k}^{\mathrm{SHP}} \bigsqcup_{x}
\end{aligned}
$$

for $x \in M$, with $k \geq 2$.

We add a few observations to this definition.

$(\alpha)$ One can likewise define the first balanced derivative of $\omega\left(: \phi^{2}:_{\text {SHP }}(x)\right)$, but since $W_{\omega, k}^{\mathrm{SHP}}$ is symmetric, its first balanced derivative vanishes. This is similar to the property of balanced derivatives of the Wick-square of the quantized linear scalar field $\phi_{o}$ on Minkowski spacetime, which can be traced back to the symmetry of

$$
\omega_{o}\left(\phi_{o}(y) \phi_{o}\left(y^{\prime}\right)\right)-\omega^{\operatorname{vac}}\left(\phi_{o}(y) \phi_{o}\left(y^{\prime}\right)\right)
$$

with respect to $y$ and $y^{\prime}$, for each quasifree Hadamard state $\omega_{o}$ of $\phi_{o}$. This provides motivation why we define the Wick-ordering by subtraction of the symmetrized Hadamard parametrix.

( $\beta$ ) Actually, $W_{\omega, k}^{\mathrm{SHP}}\left(y, y^{\prime}\right)$ and $\nabla_{a} \nabla_{b^{\prime}} W_{\omega, k}^{\mathrm{SHP}}\left(y, y^{\prime}\right)$ depend on the time-function $t$ entering into the definition of $G_{k, \epsilon}$, but for $k \geq 2$, this dependence vanishes in the coincidence limit $y=x=y^{\prime}$. Similarly, $W_{\omega, k}^{\text {SHP }}\left\lfloor_{x}\right.$ and $\nabla_{a} \nabla_{b^{\prime}} W_{\omega, k}^{\text {SHP }}\left\lfloor_{x}\right.$ are independent of $k$ for $k \geq 2$.

$(\gamma)$ One purpose of using the point-split renormalization by subtraction of the symmetrized Hadamard parametrix $\widetilde{\mathscr{G}}_{k}$ is that the latter is a locally constructed geometric quantity which is state independent, so that $: \phi^{2}:{ }_{\text {SHP }}$ and $\varlimsup_{a b}: \phi^{2}:{ }_{\text {SHP }}$ become local, covariant fields. If one applies this technique to the linear scalar field $\phi_{o}$ on Minkowski spacetime, one finds that $: \phi_{o}{ }^{2}:{ }_{\text {SHP }}$ and $\partial_{\mu \nu}: \phi_{o}{ }^{2}{ }_{\text {SHP }}$ deviate for $m>0$ from the usual flat-space definitions of $: \phi_{o}{ }^{2}:$ and $\widetilde{\partial}_{\mu \nu}: \phi_{o}{ }^{2}:$, described above, by constants. This deviation actually reflects the fact that the conventional normal-ordering procedure on Minkowski spacetime does not give rise to covariant Wick powers. This is related to the fact that the argument of the logarithm in the definition of the Hadamard parametrix, equation (9), can be chosen as $\frac{\sigma}{l^{2}}$ where $l$ is a length-scale. Our definition of the $\widetilde{\mathscr{G}}_{k}$ can be seen as the choice $l=1$ and using 
the expression for $\mathscr{W}_{2}^{\omega^{\text {vac }}}-\widetilde{\mathscr{G}}_{k}(k \geq 2)$, one can calculate that, for each Hadamard state $\omega_{o}$ of $\phi_{o}$, one has

$$
\begin{aligned}
\omega_{o}\left(: \phi_{o}{ }^{2}: \operatorname{SHP}\left(x_{o}\right)\right) & =\omega_{o}\left(: \phi_{o}^{2}:\left(x_{o}\right)\right)+c_{0, m}, \\
\omega_{o}\left(\check{\partial}_{\mu \nu}: \phi_{o}{ }^{2}: \operatorname{SHP}\left(x_{o}\right)\right) & =\omega_{o}\left(\widetilde{\partial}_{\mu \nu}: \phi_{o}{ }^{2}:\left(x_{o}\right)\right)+c_{2, m} \eta_{\mu \nu},
\end{aligned}
$$

at all points in Minkowski spacetime (details in Appendix B, see also [26] and [29] for a discussions of the relations between local covariance and the appearance of $l$ ). Here, $m$ is the mass parameter of the linear scalar field, and $\eta_{\mu \nu}$ is the Minkowski metric. The constants $c_{0, m}$ and $c_{2, m}$ vanish for $m=0$; for $m>0$, they are given by

$$
\begin{aligned}
& c_{0, m}=\frac{m^{2}}{(4 \pi)^{2}}\left[\ln \left(\frac{\mathrm{e}^{2 \gamma} m^{2}}{4}\right)-1\right], \\
& c_{2, m}=-\frac{m^{4}}{(4 \pi)^{2}}\left[\ln \left(\frac{\mathrm{e}^{2 \gamma} m^{2}}{4}\right)-\frac{5}{2}\right],
\end{aligned}
$$

where $\gamma$ denotes the Euler-Mascheroni constant. This needs to be taken into account in the definition of thermal equilibrium states below.

We can now define the concept of a local thermal equilibrium state on a globally hyperbolic curved spacetime $(M, g)$. Let $e=\left(e_{0}, e_{1}, e_{2}, e_{3}\right)$ be an orthonormal tetrad at $x \in M$, with $e_{0}$ timelike and future-pointing. Then $e$ induces an identification of $T_{x} M$ with Minkowski spacetime $M_{o}$, whereupon $e$ is identified with a basis of $M_{o}$, again with $e_{0}$ timelike and future-pointing in Minkowski spacetime. This identification is used in the following definition.

Definition 2.3. Let $\omega$ be a state with two-point function of Hadamard form for the quantized linear scalar field $\phi$ on a globally hyperbolic spacetime $(M, g)$. Then let $\phi_{o}$ denote the quantized linear scalar field, with the same parameters as $\phi$, on Minkowski spacetime $M_{o}$.

(a) We say that $\omega$ is $S_{x}^{(2)}$-thermal at a point $x \in M$ if, with some orthonormal tetrad $e=\left(e_{0}, e_{1}, e_{2}, e_{3}\right)$ at $x$ such that $e_{0}$ is timelike and future-pointing, there is a thermal equilibrium state $\omega_{o}^{\beta e}$ of $\phi_{o}$ so that - upon identification of $e$ with a basis tetrad of $M_{o}$ - the equalities

$$
\begin{aligned}
& \omega\left(: \phi^{2}: \operatorname{SHP}(x)\right)=\omega_{o}^{\beta e}\left(: \phi_{o}{ }^{2}: \operatorname{SHP}\left(x_{o}\right)\right) \\
& =\omega_{o}^{\beta e}\left(: \phi_{o}^{2}:\left(x_{o}\right)\right)+c_{0, m}, \\
& v^{a} w^{b} \omega\left(\varlimsup_{a b}: \phi^{2}: \operatorname{SHP}(x)\right)=v^{\mu} w^{\nu} \omega_{o}^{\beta e}\left(\varlimsup_{\mu \nu}: \phi_{o}{ }^{2}: \operatorname{SHP}\left(x_{o}\right)\right) \\
& =v^{\mu} w^{\nu} \omega_{o}^{\beta e}\left(\check{\partial}_{\mu \nu}: \phi_{o}^{2}:\left(x_{o}\right)\right)+c_{2, m} v^{\mu} w^{\nu} \eta_{\mu \nu}
\end{aligned}
$$

hold for all (spacelike) vectors $v, w \in T_{x} M$ with coordinates $v^{\mu} e_{\mu}=v, w^{\nu} e_{\nu}=w$, for some $x_{o} \in M_{o}$. (By translation-invariance of $\omega_{o}^{\beta e}$, the particular choice of $x_{o}$ is irrelevant.)

(b) Let $N$ be a subset of $M$. We say that $\omega$ is $S_{N}^{(2)}$-thermal if $\omega\left(: \phi^{2}:{ }_{\operatorname{SHP}}(x)\right)$ and $\omega\left(\mho_{a b}: \phi^{2}:{ }_{\text {SHP }}(x)\right)$ are continuous in $x \in N$ and if, for each $x \in N, \omega$ is $S_{x}^{(2)}$-thermal at $x$. 
The definition of $S_{x}^{(2)}$-thermal states demands the coincidence of expectation values of the SHP Wick square of $\phi$ and its balanced derivatives up to second order with the thermal equilibrium situation in flat spacetime. This amounts to saying that $S_{x}^{(2)}$ consists of linear combinations of the unit operator 1 and of the quadratic forms : $\phi^{2}$ S SHP $_{\text {S }}(x)$ and $\breve{\partial}_{a b}: \phi^{2}:_{\text {SHP }}(x)$ whose evaluations (i.e. expectation values) on states $\omega$ are given by (16) and (17). Thus, for the linear scalar field on Minkowski spacetime, $S_{x}^{(2)}$ is a small subset of $S_{x}$, and thus an $S_{x}^{(2)}$-thermal state fulfills less constraints on its thermal properties than an $S_{x}$-thermal state. We shall not follow up that matter at this point. Our definition of $S_{x}^{(2)}$-thermal states (or $S_{\mathcal{O}}^{(2)}$-thermal states) turns out to be sufficient to derive quantum energy inequalities.

Given an $S_{x}^{(2)}$-thermal state $\omega$, we shall now use the abbreviations

$$
\begin{aligned}
\vartheta^{\omega}(x) & =\omega\left(: \phi^{2}: \operatorname{SHP}(x)\right), \\
\varepsilon_{a b}^{\omega}(x) & =-\frac{1}{4} \omega\left(\varpi_{a b}: \phi^{2}:{ }_{\mathrm{SHP}}(x)\right) .
\end{aligned}
$$

Then we have

Lemma 2.4. Let $\phi$ be a linear scalar field on $(M, g)$, with mass parameter $m$, and let $\omega$ be an $S_{x}^{(2)}$-thermal state $(x \in M)$, satisfying (22) and (23) for some $\beta>0$ and an orthonormal tetrad $e=\left(e_{0}, e_{1}, e_{2}, e_{3}\right)$ at $x$. Then the following statements hold.

(a) $\vartheta^{\omega}(x)=\frac{1}{\beta^{2}} \chi_{0, m}(\beta)+c_{0, m}$, with

$$
\chi_{0, m}(\beta)=\frac{1}{2 \pi^{2}} \int_{0}^{\infty} \frac{\rho^{2} d \rho}{\left(\mathrm{e}^{\sqrt{\rho^{2}+\beta^{2} m^{2}}}-1\right) \sqrt{\rho^{2}+\beta^{2} m^{2}}} .
$$

(b) $\varepsilon_{a}^{\omega a}(x)=m^{2} \chi_{0, m}(\beta)-c_{2, m}$

(c) Suppose that $v$ is a lightlike vector at $x, v_{a} v^{a}=0$, or a timelike vector at $x$ with unit proper length, $v_{a} v^{a}=1$, and set $v^{0}=\left(e_{0}\right)_{a} v^{a}$. Then one has the bound

$$
\zeta(4) \frac{6\left(v^{0}\right)^{2}}{\pi^{2} \beta^{4}}-v_{a} v^{a} \frac{c_{2, m}}{4} \geq v^{a} v^{b} \varepsilon_{a b}(x) \geq \frac{\left(v^{0}\right)^{2}}{\beta^{4}} \chi_{2, m}(\beta)-v_{a} v^{a} \frac{c_{2, m}}{4}
$$

where

$$
\chi_{2, m}(\beta)=\frac{1}{2 \pi^{2}} \int_{0}^{\infty} \frac{\rho^{2} \sqrt{\rho^{2}+\beta^{2} m^{2}}}{\mathrm{e}^{\sqrt{\rho^{2}+\beta^{2} m^{2}}}-1} d \rho
$$

$(\zeta(4)$ is the value of the $\zeta$-function at 4 .)

Proof. The proof is based on the fact that, with respect to coordinates induced by the basis tetrad $e$

$$
\begin{aligned}
\omega_{o}^{\beta e}\left(: \phi_{o}{ }^{2}:\left(x_{o}\right)\right) & =\frac{1}{(2 \pi)^{3}} \int_{\mathbb{R}^{3}} \frac{1}{\left(\mathrm{e}^{\beta p_{0}}-1\right) p_{0}} d^{3} \underline{p}, \\
-\frac{1}{4} \partial_{\mu \nu} \omega_{o}^{\beta e}\left(: \phi_{o}{ }^{2}:\left(x_{o}\right)\right) & =\frac{1}{(2 \pi)^{3}} \int_{\mathbb{R}^{3}} \frac{p_{\mu} p_{\nu}}{\left(\mathrm{e}^{\beta p_{0}}-1\right) p_{0}} d^{3} \underline{p},
\end{aligned}
$$


where $\left(p_{\mu}\right)_{\mu=0, \ldots, 3}=\left(p_{0}, \underline{p}\right)$ and $p_{0}=\sqrt{|\underline{\mid}|^{2}+m^{2}}$ in the integrals. The stated relations then basically result from transforming the integrals into spherical polar coordinates. For the upper bound in (26) notice that the integrand is given by

$$
\left(v^{0} \sqrt{|\underline{\mid \underline{ }}|^{2}+m^{2}}-\underline{v} \cdot \underline{p}\right)^{2} /\left(\left(\mathrm{e}^{\beta \sqrt{|\underline{p}|^{2}+m^{2}}}-1\right) \sqrt{|\underline{\mid \underline{ }}|^{2}+m^{2}}\right)
$$

which is bounded above by

$$
|\underline{p}|\left(v^{0}-\frac{\underline{v} \cdot \underline{p}}{\sqrt{|\underline{p}|^{2}+m^{2}}}\right)^{2} /\left(\mathrm{e}^{\beta|\underline{\mathrm{p}}|}-1\right) .
$$

Upon integration over $p$, this can be bounded by the integrand $2\left(v^{0}\right)^{2}|p| /\left(\mathrm{e}^{\beta \mid \underline{p}} \mid-1\right)$, using that $\left(v^{0}\right)^{2}-|\underline{v}|^{2}=1$ in the timelike case and $\left(v^{0}\right)^{2}-|\underline{v}|^{2}=0$ in the lightlike case.

Now we introduce a set of states whose local temperature is bounded above by some fixed value.

Definition 2.5. Let $\beta^{\prime}>0, x \in M$. Then we define $\mathcal{L}_{\beta^{\prime}}(x)$ as the set of all $S_{x}^{(2)}$ thermal states $\omega$ of the linear scalar field on $(M, g)$ so that

$$
\vartheta^{\omega}(x) \leq \frac{1}{\left(\beta^{\prime}\right)^{2}} \chi_{0, m}\left(\beta^{\prime}\right)+c_{0, m} .
$$

If $N \subset M$, we define $\mathcal{L}_{\beta^{\prime}}(N)$ as the set of all $S_{N}^{(2)}$-thermal states of the linear scalar field on $(M, g)$ so that (28) is fulfilled for all $x \in N$.

In other words, $\omega$ is in $\mathcal{L}_{\beta^{\prime}}(x)$ if the relations (22) and (23) are fulfilled for $1 / \beta<1 / \beta^{\prime}$.

Now let $N$ be an open subset of $M$, and let $\gamma:\left[\tau_{0}, \tau_{1}\right] \rightarrow N, \tau \mapsto \gamma(\tau)$ be a geodesic with affine parameter $\tau$, and denote by $v^{a}=\dot{\gamma}^{a}$ the tangent vector field of $\gamma$. By the geodesic equation, it holds that

$$
\left(v^{a} v^{b} \nabla_{a} \nabla_{b} \vartheta^{\omega}\right)(\gamma(\tau))=\frac{d^{2}}{d \tau^{2}} \vartheta^{\omega}(\gamma(\tau))
$$

Consequently, we obtain for $\omega \in \mathcal{L}_{\beta^{\prime}}(N)$ and $\eta \in C_{0}^{2}\left(\left(\tau_{0}, \tau_{1}\right)\right)$,

$$
\begin{aligned}
\left|\int \eta(\tau)\left(v^{a} v^{b} \nabla_{a} \nabla_{b} \vartheta^{\omega}\right)(\gamma(\tau)) d \tau\right| & =\left|\int \eta^{\prime \prime}(\tau) \vartheta^{\omega}(\gamma(\tau)) d \tau\right| \\
& \leq\left\|\eta^{\prime \prime}\right\|_{L^{1}}\left|\frac{1}{\left(\beta^{\prime}\right)^{2}} \chi_{0, m}\left(\beta^{\prime}\right)+c_{0, m}\right| .
\end{aligned}
$$

Here, $\eta^{\prime \prime}$ is the second derivative of $\eta$. 


\section{Quantum energy inequalities}

Let $(M, g)$ be a globally hyperbolic spacetime, and let $\varphi$ be the classical linear scalar field on $(M, g)$ with mass parameter $m \geq 0$ and conformal coupling parameter $\xi$. If $\varphi$ is a field configuration, i.e. a smooth solution to the field equation (4), then the corresponding classical stress-energy tensor is a $\left(\begin{array}{l}0 \\ 2\end{array}\right)$ co-tensor field $T_{a b}^{(\varphi)}$ given by

$$
\begin{aligned}
T_{a b}^{(\varphi)}(x)= & \left(\nabla_{a} \varphi(x)\right)\left(\nabla_{b} \varphi(x)\right)+\frac{1}{2} g_{a b}(x)\left(m^{2} \varphi^{2}(x)-\left(\nabla^{c} \varphi\right)\left(\nabla_{c} \varphi\right)(x)\right) \\
& +\xi\left(g_{a b}(x) \nabla^{c} \nabla_{c}-\nabla_{a} \nabla_{b}-G_{a b}(x)\right) \varphi^{2}(x), \quad x \in M,
\end{aligned}
$$

where $G_{a b}=R_{a b}-\frac{1}{2} g_{a b} R$ is the Einstein tensor.

Now let $\phi$ be the quantized linear scalar field on $(M, g)$, corresponding to the choice of parameters $m$ and $\xi$. The definition of the renormalized expectation value of products and derivatives for the quantized linear scalar field $\phi$ in a state $\omega$ having two-point function of Hadamard form proceeds, similarly to what was done in the previous chapter, by point-splitting and subtraction of the SHP (see $[39,41]$ ). To this end, we define:

$$
\begin{aligned}
& \omega\left(: \phi \nabla_{a} \phi: \operatorname{SHP}(x)\right)=\nabla_{a^{\prime}} W_{\omega, k}^{\mathrm{SHP}} \bigsqcup_{x} \\
& \omega\left(: \phi \nabla_{a} \nabla_{b} \phi: \mathrm{SHP}_{\mathrm{SH}}(x)\right)=\nabla_{a^{\prime}} \nabla_{b^{\prime}} W_{\omega, k}^{\mathrm{SHP}}\left\lfloor_{x}\right. \\
& \omega\left(:\left(\nabla_{a} \phi\right)\left(\nabla_{b} \phi\right): \mathrm{SHP}(x)\right)=\nabla_{a} \nabla_{b^{\prime}} W_{\omega, k}^{\mathrm{SHP}}\left\lfloor_{x}\right.
\end{aligned}
$$

with $k \geq 2, x \in M$. (Note again that $a$ and $a^{\prime}$ are identified upon taking the coincidence limit $y=x=y^{\prime}$ on the right hand side of each equation.) Owing to the symmetry of $W_{\omega, k}^{\mathrm{SHP}}(k \geq 2)$, one can easily check that the following Leibniz rule is fulfilled for SHP Wick-products involving derivatives:

$$
\begin{aligned}
& \omega\left(\nabla_{a}\left(: \phi^{2}: \operatorname{SHP}(x)\right)\right)=2 \omega\left(: \phi \nabla_{a} \phi:_{\operatorname{SHP}}(x)\right), \\
& \omega\left(\nabla_{a}\left(: \phi \nabla_{b} \phi: \mathrm{SHP}_{\mathrm{SP}}(x)\right)\right)=\omega\left(:\left(\nabla_{a} \phi\right)\left(\nabla_{b} \phi\right): \mathrm{SHP}_{\mathrm{SP}}(x)\right)+\omega\left(: \phi \nabla_{a} \nabla_{b} \phi: \operatorname{SHP}(x)\right) .
\end{aligned}
$$

The renormalized expectation value of stress-energy is then obtained via replacing the classical expressions $\varphi^{2}(x),\left(\nabla_{a} \varphi(x)\right)\left(\nabla_{b} \varphi(x)\right)$, and so on, by $\omega\left(: \phi^{2}\right.$ : $\left._{\text {SHP }}(x)\right)$, $\omega\left(:\left(\nabla_{a} \phi\right)\left(\nabla_{b} \phi\right):\right.$ SHP $\left.(x)\right)$, etc. Using also the Leibniz rule for SHP Wick products, this leads to

$$
\begin{aligned}
\omega\left(T_{a b}^{\mathrm{SHP}}(x)\right)= & \omega\left(-: \phi \nabla_{a} \nabla_{b} \phi: \mathrm{SHP}\right. \\
& +\left(\frac{1}{4}-\xi\right) \omega\left(\nabla_{a} \nabla_{b}: \phi^{2}: \frac{1}{4} \nabla_{a} \nabla_{b}: \phi^{2}: \operatorname{SHP}(x)-g_{a b}(x) \nabla^{c} \nabla_{c}: \phi^{2}: \operatorname{SHP}(x)\right) \\
& +\frac{1}{2} g_{a b}(x) \omega\left(: \phi \nabla^{c} \nabla_{c} \phi: \mathrm{SHP}_{\mathrm{SH}}(x)+m^{2}: \phi^{2}: \operatorname{SHP}(x)\right) \\
& -\xi G_{a b}(x) \omega\left(: \phi^{2}: \operatorname{SHP}(x)\right)
\end{aligned}
$$


This expression, however, has the defect of a non-vanishing divergence. The way to cope with this problem, following Wald [39,41], is like this: It can be shown that $\nabla^{a} \omega\left(T_{a b}^{\mathrm{SHP}}(x)\right)=\nabla_{b} Q(x)^{4}$ where (apart from a free constant which can be set to a preferred value depending on the mass parameter $m) Q$ is a function which is determined by the local geometry of $(M, g)$; in particular, $Q$ is independent of the state $\omega$. One may therefore subtract the term $Q(x) g_{a b}(x)$ from $\omega\left(T_{a b}^{\mathrm{SHP}}(x)\right)$ to make the resulting quantity have vanishing divergence. There remains an ambiguity in that one may still add other $\left(\begin{array}{l}0 \\ 2\end{array}\right)$ co-tensor fields $C_{a b}$ which are determined by the local geometry of $(M, g)$ and have vanishing divergence. We take here the same view as put forward in [18], namely that the specification of $C_{a b}$ is a further datum of the underlying quantum field $\phi$ on $(M, g)$, in addition to the parameters $m$ and $\xi$. An alternative, elegant method has been proposed by Moretti [29], which nevertheless we won't follow here mainly because we would like to maintain close contact to other works on quantum energy inequalities. This understood, we finally define the renormalized expectation value of the stress energy tensor in some state $\omega$ (with two-point function of Hadamard form) of the linear scalar field $\phi$ on $(M, g)$ as

$$
\omega\left(T_{a b}^{r e n}(x)\right)=\omega\left(T_{a b}^{\mathrm{SHP}}(x)\right)-Q(x) g_{a b}(x)+C_{a b}(x), \quad x \in M .
$$

Again note that $Q$ and $C_{a b}$ are state-independent and constructed locally out of the spacetime metric $g=g_{a b}$.

Let us next observe that, for each state $\omega$ of $\phi$ with two-point function of Hadamard form, and with $R=R_{a}^{a}$ denoting the scalar curvature,

$$
\begin{aligned}
F(x) & =\omega\left(: \phi\left(\nabla^{a} \nabla_{a}+m^{2}+\xi R\right) \phi:_{\mathrm{SHP}}(x)\right) \\
& =\omega\left(: \phi\left(\nabla^{a} \nabla_{a} \phi\right):{ }_{\mathrm{SHP}}(x)\right)+\left(m^{2}+\xi R(x)\right) \omega\left(: \phi^{2}:{ }_{\mathrm{SHP}}(x)\right)
\end{aligned}
$$

is a continuous function of $x \in M$, independent of the state $\omega$, entirely determined by the local geometry of $(M, g)$ and the parameters $m$ and $\xi$ of $\phi$. To see this, note that

$$
\omega\left(: \phi\left(\nabla^{a} \nabla_{a}+m^{2}+\xi R\right) \phi:_{\mathrm{SHP}}(x)\right)=\left(\nabla^{a^{\prime}} \nabla_{a^{\prime}}+m^{2}+\xi R\right) W_{\omega, k}^{\mathrm{SHP}}\left\lfloor_{x} .\right.
$$

On the other hand, $W_{\omega, k}^{\mathrm{sHP}}\left(y, y^{\prime}\right)$ is the integral kernel of $\mathscr{W}_{\omega, k}^{\mathrm{SHP}}=\mathscr{W}_{2}^{\omega}-\widetilde{\mathscr{G}}_{k}(k \geq 2)$, and since $\mathscr{W}_{2}^{\omega}\left(f,\left(\nabla^{b} \nabla_{b}+m^{2}+\xi R\right) h\right)=0$, it follows that $\left(\nabla^{a^{\prime}} \nabla_{a^{\prime}}+m^{2}+\xi R\right) W_{\omega, k}^{\mathrm{SHP}}$ is independent of $\omega$ as the $\omega$-dependent part of $W_{\omega, k}^{\text {SHP }}$ is annihilated by the waveoperator $\left(\nabla^{a^{\prime}} \nabla_{a^{\prime}}+m^{2}+\xi R\right)$. In consequence, $F(x)=\left(\nabla^{a^{\prime}} \nabla_{a^{\prime}}+m^{2}+\xi R\right) W_{\omega, k}^{\mathrm{SHP}}\left\lfloor_{x}\right.$ is state-independent, continuous in $x$, and actually it is determined by the local geometry of $(M, g)$ since so is $\widetilde{\mathscr{G}}_{k}$ (by the Hadamard recursion relations). An explicit calculation using this relations gives the relation [29]

$$
F=3 Q
$$

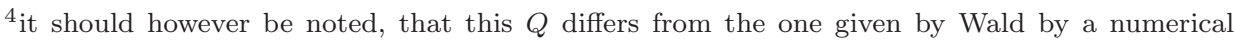
factor, which is due to the fact that we define the Wick squares using a symmetric Hadamard Parametrix which does not fulfill the wave equation in any of its two arguments, whereas Wald uses an asymmetric one which fulfills the wave equation in the first argument 

follows:

Using the Leibniz rule, we can now rewrite the expression for $\omega\left(T_{a b}^{r e n}\right)$ as

$$
\begin{aligned}
\omega\left(T_{a b}^{r e n}(x)\right)= & \omega\left(-: \phi \nabla_{a} \nabla_{b} \phi: \mathrm{SHP}\right. \\
& +\left(\frac{1}{4}-\xi\right) \omega\left(\nabla_{a} \nabla_{b}: \phi^{2}:_{\mathrm{SHP}}(x)\right) \\
& +(4 \xi-1)\left(\omega\left(-: \phi \nabla^{c} \nabla_{c} \phi \nabla_{b}: \phi^{2}:_{\mathrm{SHP}}(x)+\frac{1}{4} \nabla^{c} \nabla_{c}: \phi^{2}:_{\mathrm{SHP}}(x)\right)\right) g_{a b} \\
& +\left(\left((1-4 \xi)\left(m^{2}+\xi R\right)-\frac{1}{2} \xi R\right) g_{a b}-\xi G_{a b}\right) \omega\left(: \phi^{2}:_{\mathrm{SHP}}(x)\right) \\
& +\left(12 \xi-\frac{5}{2}\right) Q(x) g_{a b}(x)+C_{a b}(x)
\end{aligned}
$$

By (15) and once more the Leibniz rule, $\epsilon_{a b}$ can also be expressed as

$$
\epsilon_{a b}=\omega\left(-: \phi \nabla_{a} \nabla_{b} \phi: \mathrm{SHP}(x)+\frac{1}{4} \nabla_{a} \nabla_{b}: \phi^{2}: \mathrm{SHP}(x)\right)
$$

Thus, if $\omega$ is an $S_{x}^{(2)}$-thermal state of $\phi$, we obtain

$$
\begin{aligned}
\omega\left(T_{a b}^{r e n}(x)\right)= & \varepsilon_{a b}^{\omega}(x)+(4 \xi-1) g_{a b}(x) \varepsilon_{c}^{\omega c}(x) \\
& +\left(\frac{1}{4}-\xi\right) \nabla_{a} \nabla_{b} \vartheta^{\omega}(x)+\left(g_{a b}(x) \psi(x)-\xi R_{a b}(x)\right) \vartheta^{\omega}(x) \\
& +(12 \xi-5 / 2) Q(x) g_{a b}(x)+C_{a b}(x),
\end{aligned}
$$

where we use the abbreviation

$$
\psi(x)=(1-4 \xi)\left(m^{2}+\xi R(x)\right) .
$$

With this expression, we are now in the position to derive bounds on $v^{a} v^{b} \omega\left(T_{a b}^{r e n}\right)$ for lightlike or timelike vectors $v$. We will treat lower bounds first.

Theorem 3.1. Let $\phi$ be the quantized linear scalar field on $(M, g)$, with parameters $m, \xi$ and $C_{a b}$, and let $\omega$ be a state of $\phi$ having two-point function of Hadamard form.

(a) Suppose that $\xi=1 / 4$, and let $v$ be a lightlike vector at $x \in M$, or a timelike vector at $x$ with $v_{a} v^{a}=1$. If $\omega$ is in $\mathcal{L}_{\beta^{\prime}}(x), \beta^{\prime}>0$, then

$$
v^{a} v^{b} \omega\left(T_{a b}^{r e n}(x)\right) \geq q\left(x, v ; \beta^{\prime}\right)
$$

where

$$
\begin{aligned}
q\left(x, v ; \beta^{\prime}\right) & =-\frac{1}{4}\left|v^{a} v^{b} R_{a b}(x)\right|\left|\frac{1}{{\beta^{\prime}}^{2}} \chi_{0, m}\left(\beta^{\prime}\right)+c_{0, m}\right| \\
& +\left(\frac{3}{2} Q(x)-\frac{1}{4} c_{2, m}\right) v_{a} v^{a}+v^{a} v^{b} C_{a b} .
\end{aligned}
$$


(b) Let $\xi$ be arbitrary, let $N \subset M$, and let $\gamma:\left[\tau_{0}, \tau_{1}\right] \rightarrow N$ be an affinely parametrized lightlike geodesic defined on a finite interval, with tangent vector field $v^{a}=\dot{\gamma}^{a}$. Suppose that $\eta$ is in $C_{0}^{2}\left(\left(\tau_{0}, \tau_{1}\right)\right)$ with $\eta \geq 0$. If $\omega \in \mathcal{L}_{\beta^{\prime}}(N)$, there holds the bound

$$
\int \eta(\tau) v^{a} v^{b} \omega\left(T_{a b}^{r e n}(\gamma(\tau))\right) d \tau \geq q_{0}\left(\gamma, \eta ; \beta^{\prime}\right)
$$

Here, writing

$$
R_{[\gamma]}=\max _{\tau \in\left[\tau_{0}, \tau_{1}\right]}\left|\dot{\gamma}^{a}(\tau) \dot{\gamma}^{b}(\tau) R_{a b}(\gamma(\tau))\right|,
$$

and defining $C_{[\gamma]}$ analogously, the bounding constant is given by

$$
\begin{aligned}
q_{0}\left(\gamma, \eta ; \beta^{\prime}\right)= & -\left[|\xi| R_{[\gamma]}\left|\frac{1}{\beta^{\prime 2}} \chi_{0, m}\left(\beta^{\prime}\right)+c_{0, m}\right|+C_{[\gamma]}\right]\|\eta\|_{L^{1}} \\
& -\left|\frac{1}{4}-\xi\right| \cdot\left|\frac{1}{{\beta^{\prime}}^{2}} \chi_{0, m}\left(\beta^{\prime}\right)+c_{0, m}\right| \cdot\left\|\eta^{\prime \prime}\right\|_{L^{1}}
\end{aligned}
$$

(c) Let $\xi$ be arbitrary, let $N \subset M$, and let $\gamma:\left[\tau_{0}, \tau_{1}\right] \rightarrow N$ be an affinely parametrized timelike geodesic with tangent vector field $v^{a}=\dot{\gamma}^{a}$, so that $v^{a} v_{a}=1$. Assume that $\eta$ is in $C_{0}^{2}\left(\left(\tau_{0}, \tau_{1}\right)\right)$ with $\eta \geq 0$. If $\omega \in \mathcal{L}_{\beta^{\prime}}(N)$, there holds the bound

$$
\int \eta(\tau) v^{a} v^{b} \omega\left(T_{a b}^{r e n}(\gamma(\tau))\right) d \tau \geq q_{1}\left(\gamma, \eta ; \beta^{\prime}\right)
$$

where, using the notation $\psi_{[\gamma]}=\max _{\tau \in\left[\tau_{0}, \tau_{1}\right]}|\psi(\gamma(\tau))|$, and defining $Q_{[\gamma]}$ similarly, the bounding constant is given by

$$
\begin{aligned}
q_{1}\left(\gamma, \eta ; \beta^{\prime}\right)= & -\left.\left|\frac{1}{4}-\xi\right| \cdot\left|\frac{1}{{\beta^{\prime}}^{2}} \chi_{0, m}\left(\beta^{\prime}\right)+c_{0, m}\right| \cdot|| \eta^{\prime \prime}\right|_{L^{1}} \\
& -\left.\left(\psi_{[\gamma]}+|\xi| R_{[\gamma]}\right) \cdot\left|\frac{1}{{\beta^{\prime}}^{2}} \chi_{0, m}\left(\beta^{\prime}\right)+c_{0, m}\right| \cdot|| \eta\right|_{L^{1}} \\
& -\left(\left|12 \xi-\frac{5}{2}\right| Q_{[\gamma]}+|4 \xi-1|\left|c_{2, m}\right|+C_{[\gamma]}+\frac{\left|c_{2, m}\right|}{4}\right) \cdot \|\left.\eta\right|_{L^{1}} .
\end{aligned}
$$

Proof. The proof of the statement consists just of inserting the estimates of Lemma 2.4 and discarding manifestly positive terms, in combination with estimate (29) for the average of the second derivatives of $\vartheta^{\omega}$ along the geodesic. The term involving second derivatives of $\vartheta^{\omega}$ doesn't occur for $\xi=1 / 4$, which makes it possible to give a pointwise lower bound in this case.

The central assertion of Theorem 3.1 is that the lower bound of the energy density averaged along a causal geodesic depends only on the temperatures an LTE state attains on the geodesic, and is otherwise state-independent. The bound worsenes (shifts towards the left on the real axis) as the temperature increases, i.e. with increasing $1 / \beta^{\prime}$. This is related to the question of the sharpness of the obtained bounds. The point to notice here is that they were obtained by bounding the term $\epsilon_{a b} v^{a} v^{b}$ from below by the temperature independent term $-v^{a} v_{a}\left|c_{2}\right| / 4$. However, 
$\epsilon_{a b} v^{a} v^{b}$ grows with temperature as can be seen from (26) and the growth is (for high temperatures) with the fourth power of temperature. As the $\vartheta^{\omega}$ dependent term, which is responsible for the worsening of the bounds, grows (asymptotically) with the square of the temperature, it will be compensated for sufficiently high temperatures by the dropped term. By a more careful investigation one could therefore hope to obtain a lower bound where the temperature dependence is replaced by a dependence on the spacetime geometry and $\gamma$. Finally it should also be noted that the bounds are local covariant.

For upper bounds on the averaged energy density of LTE states, an additional state-dependence shows up: The bounds depend also on the tetrad $e$ appearing in the condition of $S_{x}^{(2)}$-thermality, Definition 2.3. In this sense, the lower bounds on the averaged energy densities of LTE states are stronger than the upper bounds. This is similar to what holds for averages of energy densities for arbitrary Hadamard states of the linear scalar field [14].

Let $x \in M$, and let $e=\left(e_{0}, \ldots, e_{3}\right)$ be an orthonormal tetrad at $x$ with $e_{0}$ timelike and future-pointing. We define $\mathcal{L}_{\beta^{\prime}}(x, e)$ as the set of all states $\omega$ in $\mathcal{L}_{\beta^{\prime}}(x)$ where the $S_{x}^{(2)}$-thermality conditions $(22)$ and $(23)$ hold with respect to the given tetrad. Similarly, let $N$ be a subset of $M$, and let $N \ni x \mapsto e(x)$, $e(x)=\left(e_{0}(x), \ldots, e_{3}(x)\right)$ be a $C^{0}$ field of orthonormal tetrads over $N$, with $e_{0}(x)$ timelike and future-pointing for all $x$. Then we define $\mathcal{L}_{\beta^{\prime}}(N, e)$ as the set of all states $\omega$ in $\mathcal{L}_{\beta^{\prime}}(N)$ such that, for each $x \in N$, $\omega$ satisfies the $S_{x}^{(2)}$-thermality conditions (22) and (23) with respect to $e=e(x)$. With these conventions, we obtain the following upper bounds on (averaged) energy densities.

Theorem 3.2. Let $\phi$ be the quantized linear scalar field on $(M, g)$, with parameters $m, \xi$ and $C_{a b}$, and let $\omega$ be a state of $\phi$ having two-point function of Hadamard form.

(a) Suppose that $\xi=1 / 4$, let $v$ be a lightlike vector at $x \in M$, or a timelike vector at $x$ with $v_{a} v^{a}=1$, If $\omega$ is in $\mathcal{L}_{\beta^{\prime}}(x, e), \beta^{\prime}>0$, then

$$
p\left(v, x ; \beta^{\prime}, e\right) \geq v^{a} v^{b} \omega\left(T_{a b}^{r e n}(x)\right)
$$

where

$$
\begin{aligned}
p\left(v, x ; \beta^{\prime}, e\right)=\zeta(4) & \frac{6\left(v^{0}\right)^{2}}{\pi^{2} \beta^{\prime}}+\frac{1}{2}\left|v^{a} v^{b} R_{a b}\right|\left|\frac{1}{{\beta^{\prime}}^{2}} \chi_{0, m}\left(\beta^{\prime}\right)+c_{0, m}\right| \\
+ & q\left(x, v ; \beta^{\prime}\right)
\end{aligned}
$$

with $v^{0}=v_{a}\left(e_{0}\right)^{a}$.

(b) Let $\xi$ be arbitrary, $N \subset M$, and let $\gamma:\left[\tau_{0}, \tau_{1}\right] \rightarrow N$ be an affinely parametrized lightlike geodesic defined on a finite interval, with tangent vector field $v^{a}=\dot{\gamma}^{a}$. Suppose that $\eta$ is in $C_{0}^{2}\left(\left(\tau_{0}, \tau_{1}\right)\right)$ with $\eta \geq 0$. If $\omega$ is in $\mathcal{L}_{\beta^{\prime}}(N, e)$, then

$$
p_{0}\left(\gamma, \eta ; \beta^{\prime}, e\right) \geq \int \eta(\tau) v^{a} v^{b} \omega\left(T_{a b}^{r e n}(\gamma(\tau))\right) d \tau
$$


where

$$
\begin{aligned}
& \quad p_{0}\left(\gamma, \eta ; \beta^{\prime}, e\right)=\frac{6 \zeta(4)}{\pi^{2}\left(\beta^{\prime}\right)^{2}}\left(v_{[\gamma]}^{0}\right)^{2}\|\eta\|_{L^{1}}+\left|q_{0}\left(\gamma, \beta^{\prime} ; \eta\right)\right| \\
& \text { with } v_{[\gamma]}^{0}=\max _{\tau \in\left[\tau_{0}, \tau_{1}\right]} \dot{\gamma}_{a}(\tau) e_{0}^{a}(\gamma(\tau)) .
\end{aligned}
$$

\section{Averaged null energy condition (ANEC)}

In this section we derive the averaged null energy condition (ANEC) for $S_{N}^{(2)}$ thermal states of the quantized linear scalar field $\phi$ on a globally hyperbolic spacetime $(M, g)$.

The ANEC on a state $\omega$ of $\phi$ demands that

$$
\liminf _{\tau_{ \pm} \rightarrow \pm \infty} \int_{\tau_{-}}^{\tau_{+}} v^{a} v^{b} \omega\left(T_{a b}^{r e n}(\gamma(\tau))\right) d \tau \geq 0
$$

for all complete lightlike geodesics $\gamma$ in $M$ with affine parameter $\tau$ and tangent $v^{a}=\dot{\gamma}^{a}$. If this condition holds, and if $(M, g)$ together with $\phi$ and $\omega$ are a solution to the semiclassical Einstein equation in the form

$$
G_{a b}(x)=8 \pi \omega\left(T_{a b}^{r e n}(x)\right), \quad x \in M,
$$

then this implies that

$$
\liminf _{\tau_{ \pm} \rightarrow \pm \infty} \int_{\tau_{-}}^{\tau_{+}} v^{a} v^{b} G_{a b}(\gamma(\tau)) d \tau \geq 0
$$

for all complete lightlike geodesics $\gamma$. (We address the issue for the semiclassical Einstein equations with an additional contribution by a classical stress-energy tensor below.) It has been shown that this weaker form of the usual pointwise null energy condition, which demands that $\ell^{a} \ell^{b} G_{a b}(x) \geq 0$ for all lightlike vectors $\ell^{a}$ at each $x \in M$, is still sufficient to reach the same conclusions with respect to singularity theorems as obtained from the pointwise null energy condition, i.e. that congruences of geodesics will focus with expansion diverging to $-\infty$ at finite affine geodesic parameter [25]. The validity of (39) is therefore of importance for the properties of the spacetime structure of solutions to the semiclassical Einstein equations.

It has been argued in [42] that condition (39) may be replaced by the following condition:

$$
\liminf _{\lambda \rightarrow 0} \int_{-\infty}^{\infty} \eta_{\lambda}(\tau) v^{a} v^{b} \omega\left(T_{a b}^{r e n}(\gamma(\tau))\right) d \tau \geq 0
$$

for any $\eta \in C_{0}^{2}(\mathbb{R}), \eta \geq 0$, with $\eta(0)>0$ and $\eta_{\lambda}(\tau)=\eta(\lambda \tau)$ for $\lambda>0$. More precisely, in [42] it has been shown that (42) and (40) imply that the expansion of a congruence of lightlike geodesics around $\gamma$ becomes singular along $\gamma$ (in the sense of diverging to $-\infty$ at a finite value of the affine parameter) unless it vanishes identically on $\gamma$. (In [42] this argument is given for half-line geodesics, but it carries over to the case at hand as will be shown in our Appendix A.) 
Now let $\omega \in S_{N}^{(2)}$, and let $\gamma$ be a complete lightlike geodesic in $N \subset M$ with affine parameter $\tau$ and tangent $v^{a}=\dot{\gamma}^{a}$. Then, from (32),

$$
v^{a} v^{b} \omega\left(T_{a b}^{r e n}\right)=v^{a} v^{b} \varepsilon_{a b}^{\omega}+\left(\frac{1}{4}-\xi\right) v^{a} v^{b} \nabla_{a} \nabla_{b} \vartheta^{\omega}-\xi v^{a} v^{b} G_{a b} \vartheta^{\omega}+v^{a} v^{b} C_{a b}
$$

holds along $\gamma$. Therefore, positivity properties of the (integrated) energy density $v^{a} v^{b} \omega\left(T_{a b}^{r e n}\right)$ depend also on the behaviour of $G_{a b}$ and $C_{a b}$. The sign of the term involving $G_{a b}$ is not known. To circumvent this difficulty, we assume that the underlying spacetime $(M, g)$ together with $\phi$ and $\omega$ are solutions to the semiclassical Einstein equations (40), since it is this situation in which the ANEC is applied to deduce (41) and the ensueing statements about focussing of lightlike geodesics. Supposing that $(M, g)$ together with $\phi$ and $\omega$ are solutions to the semiclassical Einstein equations, and also that $\omega$ is an $S_{N}^{(2)}$-thermal state, we obtain upon combination of (40) and (43) the equation

$$
v^{a} v^{b}\left[G_{a b}\left(1+8 \pi \xi \vartheta^{\omega}\right)-8 \pi C_{a b}\right]=8 \pi v^{a} v^{b}\left(\varepsilon_{a b}^{\omega}+\left(\frac{1}{4}-\xi\right) \nabla_{a} \nabla_{b} \vartheta^{\omega}\right)
$$

on $N$. In order to draw further conclusions, one must specify $C_{a b}$. We recall that $C_{a b}$ is a datum of the linear quantum field $\phi$, a priori only restricted by the requirement that $T_{a b}^{r e n}$ be a local covariant quantum field and divergence-free, thus $C_{a b}$ should be locally constructed from the spacetime metric. Following Wald [41], one can make the assumption that $C_{a b}$ have canonical dimension, which leads to the form

$$
C_{a b}=A g_{a b}+B G_{a b}+\Gamma \frac{\delta}{\delta g^{a b}} S_{1}(g)+D \frac{\delta}{\delta g^{a b}} S_{2}(g)
$$

where $S_{1}(g)=\int_{M} R^{2} d \operatorname{vol}_{g}, S_{2}(g)=\int_{M} R_{a b} R^{a b} d \operatorname{vol}_{g}$, and $\delta / \delta g^{a b}$ means functional differentiation with respect to the metric, with constants $A, B, \Gamma, D$ as remaining renormalization ambiguity for the quantum field $\phi$ (see [41] for additional discussion). For the rest of our discussion, we will simplify matters by assuming $\Gamma, D=0$.

Making these assumptions and observing that hence, $v^{a} v^{b} C_{a b}=B v^{a} v^{b} G_{a b}$ for all lightlike vectors $v^{a}$, (44) assumes on $N$ the form

$$
v^{a} v^{b} G_{a b}\left(1+8 \pi\left(\xi \vartheta^{\omega}-B\right)\right)=8 \pi v^{a} v^{b}\left(\varepsilon_{a b}^{\omega}+\left(\frac{1}{4}-\xi\right) \nabla_{a} \nabla_{b} \vartheta^{\omega}\right) .
$$

The constant $B$ is still free, and one may now try to choose $B$ in such a way that (46) entails the ANEC for all lightlike geodesics in $N \subset M$ and an as large as possible class of $S_{N}^{(2)}$-thermal states $\omega$. We will show that this is possible with different conditions on $B$ for the cases $\xi=1 / 4,0<\xi<1 / 4, \xi=0$.

Theorem 4.1. Let $(M, g)$ be a globally hyperbolic spacetime, let $\phi$ be the quantized linear scalar field on $(M, g)$, with parameters $m, \xi, C_{a b}$, where $C_{a b}=A g_{a b}+B R_{a b}$, with real constants $A, B$. 
Suppose further that $\omega$ is a quasifree Hadamard state for $\phi$, that $\omega \in S_{N}^{(2)}$ for $N \subset M$, and that $(M, g)$ together with $\phi$ and $\omega$ provides a solution to the semiclassical Einstein equation (40).

Let $\gamma$ be a complete lightlike geodesic in $N$ with affine parameter $\tau$ and tangent $v^{a}=\dot{\gamma}^{a}$, and let $\eta \in C_{0}^{2}(\mathbb{R}), \eta \geq 0$. Then

$$
\lim _{\lambda \rightarrow 0} \int_{-\infty}^{\infty} \eta(\lambda \tau) v^{a} v^{b} \omega\left(T_{a b}^{r e n}(\gamma(\tau))\right) d \tau \geq 0
$$

holds if any of the following groups of conditions is assumed:

1.) $\xi=1 / 4, B<1+2 \pi c_{0, m}$. In this case one even has

$$
v^{a} v^{b} \omega\left(T_{a b}^{r e n}(x)\right) \geq 0
$$

pointwise for all $x \in M$ and all lightlike vectors $v^{a}$ at $x$.

2.) $0<\xi<1 / 4, B \leq \xi c_{0, m}+1 /(8 \pi)$,

$$
\begin{aligned}
& \lambda \ln \left(\vartheta^{\omega}(\gamma(\tau / \lambda))\right) \rightarrow 0 \quad \text { as } \lambda \rightarrow 0 \quad \text { for almost all } \tau, \\
& \int_{s}^{r} \lambda\left|\ln \left(\vartheta^{\omega}(\gamma(\tau / \lambda))\right)\right| d \tau<k<\infty \quad \text { for small } \lambda \quad \text { and all } s<r \in \mathbb{R} .
\end{aligned}
$$

3.) $\xi=0, B<1 / 8 \pi$,

$$
\begin{aligned}
& \lambda \vartheta^{\omega}(\gamma(\tau / \lambda)) \rightarrow 0 \quad \text { as } \lambda \rightarrow 0 \text { for almost all } \tau, \\
& \int_{s}^{r} \lambda \vartheta^{\omega}(\gamma(\tau / \lambda)) d \tau<K<\infty \quad \text { for small } \lambda \text { and all } s<r \in \mathbb{R} .
\end{aligned}
$$

Remark. (a) If, instead of (40), the semiclassical Einstein equations are assumed to hold in the form

$$
G_{a b}(x)=8 \pi\left(T_{a b}^{\text {class }}(x)+\omega\left(T_{a b}^{r e n}(x)\right)\right)
$$

with a stress-energy tensor $T_{a b}^{\text {class }}$ for classical, macroscopic matter distribution, and if it is assumed that this stress-energy tensor fulfills the pointwise null energy condition $\ell^{a} \ell^{b} T_{a b}^{c l a s s}(x) \geq 0$ for all lightlike vectors $\ell^{a}$ at each point $x \in M$, then the statements of the theorem remain valid with $T_{a b}^{c l a s s}+\omega\left(T_{a b}^{r e n}\right)$ in place of $\omega\left(T_{a b}^{r e n}\right)$. (b) Conditions (48) and (49) say, roughly speaking, that $\vartheta^{\omega}(\gamma(\tau))$ should not grow faster than $\mathrm{e}^{|\tau|^{(1-\epsilon)}}$ for $|\tau| \rightarrow \infty$, while (50) and (51) say that $\vartheta^{\omega}(\gamma(\tau))$ should not grow faster than $|\tau|^{1-\epsilon}$ as $|\tau| \rightarrow \infty$. Since $\vartheta^{\omega}(\gamma(\tau))=\left(\beta(\gamma(\tau))^{-2} \chi_{0, m}(\beta(\gamma(\tau)))+\right.$ $c_{0, m}$ and since

$$
\chi_{0, m}(\beta) \rightarrow \frac{1}{2 \pi^{2}} \int_{0}^{\infty} \frac{\rho}{\mathrm{e}^{\rho}} d \rho \text { for } \beta \rightarrow 0,
$$

this means that the growth of the temperature $1 / \beta(\gamma(\tau))$ at $\gamma(\tau)$ appearing in Definition 2.3 of $S_{\gamma(\tau)}^{(2)}$-thermality $\omega$ should not exceed $\mathrm{e}^{|\tau|^{(1-\epsilon) / 2}}$ and $|\tau|^{(1-\epsilon) / 2}$ as $|\tau| \rightarrow \infty$, respectively. 
Proof of Theorem 4.1. 1.) If $\xi=1 / 4$, then (46) assumes the form

$$
v^{a} v^{b} G_{a b}\left(1+8 \pi\left(\vartheta^{\omega} / 4-B\right)\right)=8 \pi v^{a} v^{b} \varepsilon_{a b}^{\omega} .
$$

If $B<1+2 \pi c_{0, m}$, then the factor $1+8 \pi\left(\vartheta^{\omega} / 4-B\right)$ is strictly positive, as is the right hand side of (52). This equality holds pointwise at all $x \in M$ and for all lightlike vectors $v^{a}$, thus proving, in combination with the assumed property (40), the statement of the theorem.

2.) For $0<\xi<1 / 4, B=\xi c_{0, m}+1 /(8 \pi)-\xi c$, where $c \geq 0$, (46) takes the form

$$
v^{a} v^{b} G_{a b}\left(8 \pi \xi\left(\vartheta^{\omega}-c_{0, m}+c\right)\right)=8 \pi v^{a} v^{b} \varepsilon_{a b}^{\omega}+8 \pi(1 / 4-\xi) v^{a} v^{b} \nabla_{a} \nabla_{b} \vartheta^{\omega} .
$$

Observing that $v^{a} v^{b} \nabla_{a} \nabla_{b} c_{0, m}=0$, the last equation is turned into

$$
v^{a} v^{b} G_{a b}=\frac{v^{a} v^{b} \varepsilon_{a b}^{\omega}}{\xi\left(\vartheta^{\omega}-c_{0, m}+c\right)}+\frac{(1 / 4-\xi) v^{a} v^{b} \nabla_{a} \nabla_{b}\left(\vartheta^{\omega}-c_{0, m}\right)}{\xi\left(\vartheta^{\omega}-c_{0, m}+c\right)}
$$

where it was used that $\vartheta^{\omega}-c_{0, m}+c>0$. The first term on the right hand side of (54) is positive. Upon integration against a non-negative $C_{0}^{2}$ weighting function $\eta$ along the geodesic $\gamma$ we obtain, using the abbreviation

$$
u(\tau)=\vartheta^{\omega}(\gamma(\tau))-c_{0, m},
$$

the inequality

$$
\int \eta(\tau)\left(v^{a} v^{b} G_{a b}\right)(\gamma(\tau)) d \tau \geq \frac{1 / 4-\xi}{\xi} \int \eta(\tau) \frac{u^{\prime \prime}(\tau)}{u(\tau)+c} d \tau
$$

By partial integration,

$$
\int \eta(\tau) \frac{u^{\prime \prime}(\tau)}{u(\tau)+c} d \tau=\int \eta(\tau)\left(\frac{u^{\prime}(\tau)}{u(\tau)+c}\right)^{2} d \tau+\int \ln (u(\tau)+c) \eta^{\prime \prime}(\tau) d \tau .
$$

Thus, since the first integral on the right hand side is non-negative, $(1 / 4-\xi) / \xi>0$ for the $\xi$ considered and using the monotonicity of the logarithm together with $c \geq 0$,

$$
\int \eta(\lambda \tau)\left(v^{a} v^{b} G_{a b}\right)(\gamma(\tau)) d \tau \geq \frac{1 / 4-\xi}{\xi} \int \lambda \ln (u(\tau / \lambda)) \eta^{\prime \prime}(\tau) d \tau,
$$

and owing to assumptions (48) and (49), the expression on the right hand side converges to 0 as $\lambda \rightarrow 0$. Equation (47) is then again implied by the assumed property $(40)$.

3.) If $\xi=0$, equation (46) turns into

$$
v^{a} v^{b} G_{a b}(1-8 \pi B)=8 \pi v^{a} v^{b} \varepsilon_{a b}^{\omega}+\frac{1}{4} v^{a} v^{b} \nabla_{a} \nabla_{b} \vartheta^{\omega},
$$

and by the condition on $B$, the factor $1-8 \pi B$ is strictly positive. Observing again positivity of $8 \pi v^{a} v^{b} \varepsilon_{a b}^{\omega}$, upon integration against a non-negative $C_{0}^{2}$ weighting function $\eta$ along $\gamma$ one obtains

$$
\int \eta(\lambda \tau) v^{a} v^{b} G_{a b}(\gamma(\tau)) d \tau \geq \frac{1}{4(1-8 \pi B)} \int \lambda u(\tau / \lambda) \eta^{\prime \prime}(\tau) d \tau
$$


and the right hand side converges to 0 as $\lambda \rightarrow 0$ by assumptions (50) and (51). Again (47) is deduced from the assumed validity of (40).

\section{Generalized local thermal equilibrium states}

The notion of LTE states in [9], and the related definition of $S_{x}^{(2)}$-thermal states, is actually more general than the definition given in Section 2. In [9] the possibility was considered that an LTE state $\omega$ coincides on $S_{x}$-observables not necessarily with a thermal equilibrium state at sharp temperature in a certain Lorentz frame, but with a mixture of such states.

In our setting, where we work with the linear scalar field, this corresponds to a modification of Definition 2.3 as follows. As a consequence of eqn.(12), $\omega_{o}^{\beta e}$, the quasifree thermal equilibrium state with respect to the Minkowski tetrad $e=$ $\left(e_{0}, e_{1}, e_{2}, e_{3}\right)$ at inverse temperature $\beta$, depends only on $\boldsymbol{\beta}=\beta e_{0}$. This quantity completely parametrizes $\omega_{o}^{\beta e}$, so we write $\omega_{o}^{\boldsymbol{\beta}}$ in place of $\omega_{o}^{\beta e}$. The vectors $\boldsymbol{\beta}$ take values in $V^{+}$, the set of future-directed timelike vectors in Minkowski spacetime.

Let $(M, g)$ be a globally hyperbolic spacetime, let $V_{x}^{+} \subset T_{x} M$ be the set of future-directed timelike vectors at $x \in M$, and let $\rho_{x}$ be a Borel measure on $V_{x}^{+}$supported on a compact subset $B_{x} \subset V_{x}^{+}$, with $\int_{B_{x}} d \rho_{x}(\boldsymbol{\beta})=1$. Then we say that a Hadamard state $\omega$ of the linear scalar field $\phi$ on $(M, g)$ is a generalized $S_{x}^{(2)}$-thermal state if

$$
\begin{gathered}
\omega\left(: \phi^{2}:{ }_{\mathrm{SHP}}(x)\right)=\int_{B_{x}} \omega_{o}^{\boldsymbol{\beta}}\left(: \phi_{o}{ }^{2}:\left(x_{o}\right)\right) d \rho_{x}(\boldsymbol{\beta})+c_{0, m}, \\
v^{a} w^{b} \omega\left(\check{\partial}_{a b}: \phi^{2}:_{\mathrm{SHP}}(x)\right)=v^{\mu} w^{\nu} \int_{B_{x}} \omega_{o}^{\boldsymbol{\beta}}\left(\check{\partial}_{\mu \nu}: \phi_{o}{ }^{2}:\left(x_{o}\right)\right) d \rho(\boldsymbol{\beta})+c_{2, m} v^{\mu} w^{\nu} \eta_{\mu \nu}
\end{gathered}
$$

holds for all (spacelike) vectors $v, w \in T_{x} M$ for some $x_{o} \in M_{o}$. Making further the assumption that $F \mapsto \int_{M} \int_{B_{x}} F(x, \boldsymbol{\beta}) d \rho_{x}(\boldsymbol{\beta}) d \operatorname{vol}(x), F \in C_{0}^{\infty}(T M, \mathbb{C})$ is a distribution (on the manifold $T M$ ), such that $x \mapsto \int_{B_{x}} F(x, \boldsymbol{\beta}) d \rho_{x}(\boldsymbol{\beta})$ is $C^{2}$, one can define generalized $S_{N}^{(2)}$-thermal states in analogy to the definition of $S_{N}^{(2)}$ thermal states in Section 2.

With these conventions and assumptions, the results of Theorems 3.1, 3.2 and 4.1 extend to generalized $S_{N}^{(2)}$-thermal states, under identical assumptions, except that the bounds have to be corrected for the $\rho_{x}$-integrations. It should be obvious how this is to be done.

\section{Discussion and outlook}

We have generalized the concept of local thermal equilibrium states of [9], or rather, the concept of $S_{x}^{(2)}$-thermal states, to the quantized linear scalar field models on generic globally hyperbolic spacetimes, and have shown that one can derive certain quantum energy inequalities for such states. The lower bounds appearing in the quantum energy inequalities of local thermal equilibrium states depend only 
on the local temperature of the states, i.e. thermal function $\vartheta^{\omega}$, corresponding to the expectation value of the Wick-square in local thermal equilibrium states. The upper bounds, instead, depend also on the local frames with respect to which $S_{x}^{(2)}$-thermality is defined. In this sense, the lower bounds are stronger (have less dependence on the states) than the upper quantum energy inequality bounds. This is a feature also found for quantum energy inequalities of general Hadamard states of the linear scalar fields, and has led to the proposal to consider 'relative quantum energy inequalities' as a more general variant of quantum energy inequalities which has the potential to be valid also in interacting quantum field theories [18]. Moreover, the quantum energy inequalities for thermal equilibrium states are local covariant.

A major purpose of quantum energy inequalities, especially in local covariant form, is to provide information about the structure of spacetime geometries appearing as solutions to the semiclassical Einstein equations. Quite generally, they serve as stability conditions on quantum matter, and ensure that correspondingly the (semiclassical) gravitational interaction is attractive, at least when averaged over sufficiently extended spacetime regions. The averaged null energy condition which we proved for certain values of the curvature coupling $\xi$ and certain values of the renormalization constants is of a similar nature. One may also take the requirement that the ANEC should be fulfilled for suitable thermal equilibrium states as a constraining condition on the largely free choice of renormalization constants for the stress-energy tensor. Certainly a demand in this spirit leads to further relations between the renormalization constants, the parameters fixing the field model, and possibly geometrical quantities, and for this reason it is attractive to further study quantum energy inequalities and ANEC in the context of solutions to the semiclassical Einstein equations. Furthermore it might well be that the condition of local thermality has to be strengthened by fixing some renormalization freedom; in the setup chosen here this would mean imposing requirements on $C_{a b}$ but as the concept of local thermality is defined with reference to the balanced derivatives these requirements on $C_{a b}$ could then also be shifted to modified balanced derivatives.

One important issue we haven't addressed at all so far is the existence of local thermal equilibrium states, or at least $S_{N}^{(2)}$ states for subsets $N$ in spacetime. We have simply assumed that there are such local thermal equilibrium states to which our results apply. The question if there are local thermal equilibrium states in generic spacetimes is an interesting and difficult problem, for which we can't offer, as yet, any route to its solution. However, the existence of LTE states for the massless and the massive Klein-Gordon fields on (parts of) Minkowski spacetime has been established, with an interesting relation to situations resembling a big bang scenario $[8,27]$.

The question if local thermal equilibrium states exist is a first step towards the question how generic they are. One is inclined to think that within certain time- and energy scales, local thermal equilibrium states should be the archetypical physical states in the sense that, if one is asked to randomly pick a state in 
the physical state space (of a quantum field theory), then the result would be a local thermal equilibrium state with overwhelming likelihood. At least this is expected for interacting quantum field theories since interaction tends to equilibrate subsystems (or degrees of freedom) of a large system. If this turned out to be true, and if the temperature distribution of such states turned out to allow ANEC results similar to those of Theorem 4.1, then one would be led to conclude that (under general additional assumptions) the occurrence of singularities in solutions to the semiclassical Einstein equations is a generic feature. It would be of utmost interest to investigate this circle of questions further particularly in scenarios of early cosmology.

\section{Appendix A.}

A. We will present a result on real-valued solutions $\theta(t)$ of the differential equation

$$
\theta^{\prime}(t)+\mu \theta(t)^{2}=-f(t), \quad t \in \mathbb{R},
$$

where $\mu>0$ and $f \in C^{1}(\mathbb{R}, \mathbb{R})$, with initial condition

$$
\theta(0)=\theta_{0} \text {. }
$$

It follows from the Picard-Lindelöf Theorem that there is an open interval $(a, b)$ containing 0 , which may be finite, semi-finite or infinite (i.e. coinciding with $\mathbb{R}$ ), such that this interval is the domain of the unique, inextensible $C^{1}$ solution $\theta$ of (56) satisfying the initial condition. In this case, we call $\theta$ the maximal solution of (56) defined by the initial condition, and refer to $(a, b)$ as the maximal domain.

The following statement is a variation on a similar result in [42], and it uses a very similar argument, the main difference being that the assumption (58) here is slightly different from that in [42], where the integral is taken over a semi-axis. Note also that our parameter $\lambda$ corresponds to $1 / \lambda$ in the notation of [42].

Theorem A.1. Suppose that $f \in C^{1}(\mathbb{R}, \mathbb{R})$ has the property

$$
\liminf _{\lambda \rightarrow 0} \int_{-\infty}^{\infty} f(t) \eta(\lambda t) d t \geq 0
$$

for the function $\eta(t)=\left(1-t^{2}\right)^{4}$ for $|t|<1, \eta(t)=0$ for $|t| \geq 1$.

Then either the maximal domain of $\theta$ coincides with all of the real axis and $\theta(t)=0$ for all $t \in \mathbb{R}$, or the maximal domain $(a, b)$ of $\theta$ is a finite or semifinite interval. In this case, $\theta(t) \rightarrow \mp \infty$ for $t$ approaching the finite boundary at the right/left side of the maximal domain (in the finite case this holds with the respective sign for both boundaries). In particular, this is the case if $\theta\left(t_{0}\right) \neq 0$ for some $t_{0}$ in the maximal domain of $\theta$.

Proof. Consider the auxiliary differential equation

$$
u^{\prime \prime}(t)+\frac{f(t)}{\mu} u(t)=0 .
$$


For the initial values $u(0)=1, u^{\prime}(0)=\theta_{0}$ and the given $f$ this linear differential equation has by the Picard-Lindelöf Theorem a unique, global solution $u \in C^{2}(\mathbb{R}, \mathbb{R})$. Furthermore, this solution is nonzero in some neighbourhood of 0 . For points from this neighbourhood, one can then rewrite (59) as

$$
\frac{\mathrm{d}}{\mathrm{d} t}\left(\frac{u^{\prime}(t)}{u(t)}\right)+\left(\frac{u^{\prime}(t)}{u(t)}\right)^{2}=-\frac{f(t)}{u(t)}
$$

which implies that $\hat{\theta}(t) \equiv \frac{u^{\prime}(\mu t)}{u(\mu t)}$ fulfills equation (56). Furthermore, $\hat{\theta}$ also satisfies the initial condition (57) and by the uniqueness part in the Picard-Lindelöf Theorem it therefore agrees with $\theta$. This however implies that the only way in which $\theta$ can fail to be $C^{1}$ at a boundary point $c=a$ or $c=b$ of a semi-finite interval is a zero of $u$ at $\mu c$. At this zero $u^{\prime}$ has to differ from zero, otherwise $u$ as a $C^{2}$-solution to (59) with initial conditions $u(\mu c)=0, u^{\prime}(\mu c)=\lim _{x \rightarrow \mu c} u^{\prime}(x)=0$ would be identically zero in contradiction to the initial values for $u$ at 0 . By continuity, $u^{\prime}$ is therefore nonzero in a neighbourhood of $\mu c$, and by $(56), \theta(t)=\frac{u^{\prime}(\mu t)}{u(\mu t)}$ approaches the value $-\infty$ for $t \rightarrow c, t<c$ (right boundary point) or the value $+\infty$ for $t \rightarrow c, t>c$ (left boundary point). For proving that $\theta$ diverges at the boundary (boundaries) of a semi-finite interval it is therefore sufficient to show that $\theta$ cannot be continued as a $C^{1}$ function beyond this boundary.

With the definition of $\eta$ as above, and provided that the maximal domain of $\theta$ coincides with all of $\mathbb{R}$, one has for $0<\lambda<1$,

$$
\begin{aligned}
\int_{-\infty}^{\infty} \theta^{\prime}(t) \eta(\lambda t) d t & =-\int_{-\infty}^{\infty} \theta(t) \lambda \eta^{\prime}(\lambda t) d t \\
& =8 \lambda \int_{-1 / \lambda}^{1 / \lambda} \theta(t)(\lambda t)\left(1-(\lambda t)^{2}\right)^{3} d t \\
& \geq-8 \lambda \int_{-1 / \lambda}^{1 / \lambda}|\theta(t)|\left(1-(\lambda t)^{2}\right)^{2} d t
\end{aligned}
$$

owing to the fact that both $|\lambda t|$ and $\left|\left(1-(\lambda t)^{2}\right)\right|$ are bounded by 1 on the domain of integration. Combining this with (56) and (58) leads to

$$
\limsup _{\lambda \rightarrow 0}-8 \lambda \int_{-1 / \lambda}^{1 / \lambda}|\theta(t)|\left(1-(\lambda t)^{2}\right)^{2} d t+\mu \int_{-1 / \lambda}^{1 / \lambda} \theta(t)^{2}\left(1-(\lambda t)^{2}\right)^{4} d t \leq 0 .
$$

Using also the Cauchy-Schwarz inequality

$$
\int_{-1 / \lambda}^{1 / \lambda}|\theta(t)|\left(1-(\lambda t)^{2}\right)^{2} d t \leq\left(\int_{-/ \lambda}^{1 / \lambda} \theta(t)^{2}\left(1-(\lambda t)^{2}\right)^{4} d t\right)^{1 / 2}\left(\int_{-1 / \lambda}^{1 / \lambda} 1 d t\right)^{1 / 2}
$$


the estimate (60) can be replaced by

$$
\begin{aligned}
\limsup _{\lambda \rightarrow 0}-\frac{8}{\mu} \sqrt{2 \lambda}\left(\int_{-1 / \lambda}^{1 / \lambda} \theta(t)^{2}\left(1-(\lambda t)^{2}\right)^{4} d t\right)^{1 / 2} & \\
& +\int_{-1 / \lambda}^{1 / \lambda} \theta(t)^{2}\left(1-(\lambda t)^{2}\right)^{4} d t \leq 0,
\end{aligned}
$$

which shows that $\int_{-\infty}^{\infty} \theta(t)^{2} d t=0$ upon using Levi's theorem. Since $\theta$ is $C^{1}$, this implies that $\theta(t)=0$ for all $t$.

We have therefore shown that the assumption of $\theta$ being $C^{1}$ on all of $\mathbb{R}$ implies $\theta(t)=0$ for all $t \in \mathbb{R}$; if on the other hand $\theta$ is $C^{1}$ only on a maximal finite or semi-finite interval, then by the statement in the first paragraph of the proof, it will diverge at the finite boundaries of this interval in the indicated way.

B. Here we will calculate the constants $c_{0, m}, c_{2, m}$ that arise when defining the Wick-square and the second balanced derivative on Minkowski spacetime using the covariant point-split renormalization. A similar calculation can also be found in the Appendix B of [29], the different conventions adapted here however lead to small changes in some of the formulas appearing.

The Hadamard recursion-relations satisfied by the functions $U_{j}$ in (10) read with our sign-conventions:

$$
\begin{aligned}
-2\left(\nabla^{\kappa} \sigma\right) \nabla_{\kappa} U_{0}-\left(4+\nabla^{\kappa} \nabla_{\kappa} \sigma\right) U_{0} & =\left(\nabla^{\kappa} \nabla_{\kappa}+m^{2}+\xi R\right) U \\
-2\left(\nabla^{\kappa} \sigma\right) \nabla_{\kappa} U_{j+1}+\left(4 j-\nabla^{\kappa} \nabla_{\kappa} \sigma\right) U_{j+1} & =\frac{\left(\nabla^{\kappa} \nabla_{\kappa}+m^{2}+\xi R\right) U_{j}}{j+1} .
\end{aligned}
$$

For Minkowski spacetime, $U$ is identically one, $\nabla^{\kappa} \nabla_{\kappa} \sigma=-8$ and the unique solutions of the resulting recursion relations

$$
\begin{aligned}
4\left(x-x^{\prime}\right)^{\kappa} \nabla_{\kappa} U_{0}+4 U_{0} & =m^{2} \\
4\left(x-x^{\prime}\right)^{\kappa} \nabla_{\kappa} U_{j+1}+4(2+j) U_{j+1} & =\frac{\left(m^{2}+\nabla^{\kappa} \nabla_{\kappa}\right) U_{j}}{j+1}
\end{aligned}
$$

that remain bounded for $x \rightarrow x^{\prime}$ are easily calculated (e.g. using the method of characteristics) as

$$
U_{j}=\frac{1}{j !(j+1) !}\left(\frac{m^{2}}{4}\right)^{j+1} .
$$

For non-lightlike $x-x^{\prime}$ where $G_{k, \epsilon}$ is a regular distribution (the corresponding function being obtained as the pointwise limit $\epsilon \rightarrow 0$ ) we have with the abbreviation $\left(x-x^{\prime}\right)^{2}:=\eta_{a b}\left(x-x^{\prime}\right)^{a}\left(x-x^{\prime}\right)^{b}$ :

$$
G_{1,0}\left(x, x^{\prime}\right)=\frac{1}{4 \pi^{2}}\left(\frac{1}{-\left(x-x^{\prime}\right)^{2}}+\frac{m^{2}}{4} \ln \left(-\left(x-x^{\prime}\right)^{2}\right)\left[1+\frac{-m^{2}\left(x-x^{\prime}\right)^{2}}{8}\right]\right)
$$

(it will be seen in the course of the calculation, that $G_{1,0}$ is actually sufficient to calculate the second balanced derivative, one does not need $\left.G_{2,0}\right)$. The two-point 
function $W_{2}^{\omega^{\mathrm{vac}}}$ of the Minkowski vacuum state $\omega^{\mathrm{vac}}$ for spacelike $\left(x-x^{\prime}\right)$ is given by $[3]$

$$
W_{2}^{\omega^{\mathrm{vac}}}\left(x, x^{\prime}\right)=\frac{m}{4 \pi^{2}} \frac{K_{1}\left(m \sqrt{-\left(x-x^{\prime}\right)^{2}}\right)}{\sqrt{-\left(x-x^{\prime}\right)^{2}}}
$$

and using the asymptotic expansion of the modified Bessel function $K_{1}$ for small arguments, the terms up to the order $\left(x-x^{\prime}\right)^{2}$ of the two-point function are given by

$$
\begin{aligned}
W_{2}^{\omega^{\mathrm{vac}}}\left(x, x^{\prime}\right)= & \frac{1}{4 \pi^{2}}\left(\frac{1}{-\left(x-x^{\prime}\right)^{2}}+\frac{m^{2}}{4} \ln \left(\frac{-m^{2}\left(x-x^{\prime}\right)^{2}}{4}\right)\left[1-\frac{m^{2}}{8}\left(x-x^{\prime}\right)^{2}\right]\right. \\
& \left.+\frac{m^{2}}{4}\left[(2 \gamma-1)+(2 \gamma-5 / 2) \frac{-m^{2}\left(x-x^{\prime}\right)^{2}}{8}\right]\right)
\end{aligned}
$$

(here and in the following, $x-x^{\prime}$ is now assumed to be spacelike). The difference $W_{2}^{\omega^{\mathrm{vac}}}(x+\zeta, x-\zeta)-G_{1,0}(x+\zeta, x-\zeta)$ to the order required for the calculation of $\omega^{\infty}\left(: \phi^{2}: \operatorname{SHP}(x)\right)$ and $\omega^{\infty}\left(\varlimsup_{\mu \nu}: \phi^{2}: \operatorname{SHP}(x)\right)$ is then

$$
\begin{aligned}
W_{2}^{\omega^{\mathrm{vac}}}(x+\zeta, x-\zeta)-G_{1,0}(x+\zeta, x-\zeta)= & \frac{m^{2}}{(4 \pi)^{2}}\left[\ln \left(\frac{m^{2}}{4}\right)+2 \gamma-1\right. \\
& \left.+\left(\ln \left(\frac{m^{2}}{4}\right)+2 \gamma-5 / 2\right) \frac{-m^{2} \zeta^{2}}{2}\right] .
\end{aligned}
$$

With this expression one calculates

$$
\begin{gathered}
\omega^{\infty}\left(: \phi^{2}: \operatorname{SHP}(x)\right)=\frac{m^{2}}{(4 \pi)^{2}}\left[\ln \left(\frac{e^{2 \gamma} m^{2}}{4}\right)-1\right]=: c_{0, m} \\
\omega^{\infty}\left(\check{\partial}_{\mu \nu}: \phi^{2}: \operatorname{SHP}(x)\right)=-\frac{m^{4}}{(4 \pi)^{2}}\left[\ln \left(\frac{e^{2 \gamma} m^{2}}{4}\right)-\frac{5}{2}\right] \eta_{\mu \nu}=: c_{2, m} \eta_{\mu \nu}
\end{gathered}
$$

and from this one reads of the equations (18) and (19).

\section{Acknowledgements}

The authors would like to thank D. Buchholz for discussions on local thermal equilibrium states. J. Schlemmer gratefully acknowledges financial support by the International Max Planck Research School (IMPRS).

\section{References}

[1] M. Alcubierre, The Warp Drive: Hyper-Fast Travel within General Relativity, Class. Quant. Grav. 11 (1994), L73, gr-qc/0009013. 
[2] C. Bär, N. Ginoux and F. Pfäffle, Wave Equations on Lorentzian Manifolds and Quantization, ESI Lectures in Mathematics and Physics, European Mathematical Society (EMS), Zürich (2007).

[3] N. N. Bogolubov, A. A. Logunov, A. I. Oksak and I. T. Todorov, General Principles of Quantum Field Theory, vol. 10 of Mathematical Physics and Applied Mathematics, Kluwer Academic Publishers Group, Dordrecht (1990).

[4] M. Bordag, U. Mohideen and V.M. Mostepanenko, New Developments in the Casimir Effect, Phys. Rept. 353 (2001), 1.

[5] A. Borde, Geodesic Focusing, Energy Conditions and Singularities, Class. Quant. Grav. 4 (1987), 343.

[6] O. Bratteli and D.W. Robinson, Operator Algebras and Quantum Statistical Mechanics 2, Texts and Monographs in Physics, Springer-Verlag, Berlin, second edn. (1997).

[7] R. Brunetti, K. Fredenhagen and R. Verch, The Generally Covariant Locality Principle - A New Paradigm for Local Quantum Physics, Commun. Math. Phys. 237 (2003), 31, math-ph/0112041.

[8] D. Buchholz, On Hot Bangs and the Arrow of Time in Relativistic Quantum Field Theory, Commun. Math. Phys. 237 (2003), 271, hep-th/0301115.

[9] D. Buchholz, I. Ojima and H. Roos, Thermodynamic Properties of Non-Equilibrium States in Quantum Field Theory, Annals Phys. 297 (2002), 219, hep-ph/0105051.

[10] D. Buchholz and J. Schlemmer, Local Temperature in Curved Spacetime, Class. Quant. Grav. 24 (2007), F25, gr-qc/0608133.

[11] H. Epstein, V. Glaser and A. Jaffe, Nonpositivity of the Energy Density in Quantized Field Theories, Nuovo Cimento 36 (1965), 1016.

[12] C. J. Fewster, A General Worldline Quantum Inequality, Class. Quant. Grav. 17 (2000), 1897, gr-qc/9910060.

[13] C. J. Fewster, Quantum Energy Inequalities and Stability Conditions in Quantum Field Theory, (2005), math-ph/0502002.

[14] C. J. Fewster and L.W. Osterbrink, Quantum Energy Inequalities for the NonMinimally Coupled Scalar Field, J. Phys. A41 (2008), 025402, arXiv:0708.2450 $[\mathrm{gr}-\mathrm{qc}]$.

[15] C. J. Fewster and M. J. Pfenning, A Quantum Weak Energy Inequality for Spin-One Fields in Curved Spacetime, J. Math. Phys. 44 (2003), 4480, gr-qc/0303106.

[16] C. J. Fewster and T. A. Roman, Null Energy Conditions in Quantum Field Theory, Phys. Rev. D67 (2003), 044003, gr-qc/0209036.

[17] C. J. Fewster and T. A. Roman, On Wormholes with Arbitrarily Small Quantities of Exotic Matter, Phys. Rev. D72 (2005), 044023, gr-qc/0507013.

[18] C.J. Fewster and C.J. Smith, Absolute Quantum Energy Inequalities in Curved Spacetime, (2007), gr-qc/0702056.

[19] C. J. Fewster and R. Verch, A Quantum Weak Energy Inequality for Dirac Fields in Curved Spacetime, Commun. Math. Phys. 225 (2002), 331, math-ph/0105027.

[20] C. J. Fewster and R. Verch, Stability of Quantum Systems at Three Scales: Passivity, Quantum Weak Energy Inequalities and the Microlocal Spectrum Condition, Commun. Math. Phys. 240 (2003), 329, math-ph/0203010. 
[21] L.H. Ford, Quantum Coherence Effects and the Second Law of Thermodynamics, Proc. R. Soc. Lond. A 364 (1978), 227.

[22] L. H. Ford and T. A. Roman, Quantum Field Theory Constrains Traversable Wormhole Geometries, Phys. Rev. D53 (1996), 5496, gr-qc/9510071.

[23] S. A. Fulling, Nonuniqueness of Canonical Field Quantization in Riemannian SpaceTime, Phys. Rev. D7 (1973), 2850.

[24] D. Guido and R. Longo, A Converse Hawking - Unruh Effect and dS(2)/CFT Correspondence, Ann. Henri Poinc. 4 (2003), 1169.

[25] S. W. Hawking and G.F. R. Ellis, The Large Scale Structure of Space-time, Cambridge University Press, London (1973).

[26] S. Hollands and R. M. Wald, Local Wick Polynomials and Time Ordered Products of Quantum Fields in Curved Spacetime, Commun. Math. Phys. 223 (2001), 289, gr-qc/0103074.

[27] R. Hübener, Lokale Gleichgewichtszustände massiver Bosonen, Diplomarbeit, University of Göttingen (2005).

[28] B.S. Kay and R. M. Wald, Theorems on the Uniqueness and Thermal Properties of Stationary, Nonsingular, Quasifree States on Spacetimes with a Bifurcate Killing Horizon, Phys. Rept. 207 (1991), 49.

[29] V. Moretti, Comments on the Stress-Energy Tensor Operator in Curved Spacetime, Commun. Math. Phys. 232 (2003), 189, gr-qc/0109048.

[30] M.S. Morris, K.S. Thorne and U. Yurtsever, Wormholes, Time Machines, and the Weak Energy Condition, Phys. Rev. Lett. 61 (1988), 1446.

[31] M. J. Pfenning and L. H. Ford, The Unphysical Nature of 'Warp Drive', Class. Quant. Grav. 14 (1997), 1743, gr-qc/9702026.

[32] T. A. Roman, On the 'Averaged Weak Energy Condition' and Penrose's Singularity Theorem, Phys. Rev. D37 (1988), 546.

[33] T. A. Roman, Some Thoughts on Energy Conditions and Wormholes, in M. Novello, S. Perez Bergliaffa, R. Ruffini, eds., Proceedings of the MG10 Meeting, p. 1909, gr-qc/0409090.

[34] H. Sahlmann and R. Verch, Passivity and microlocal spectrum condition, Commun. Math. Phys. 214 (2000), 705, math-ph/0002021.

[35] F. Serry, D. Walliser and G. J. Maclay, The Role of the Casimir Effect in the Static Deflection and Stiction of Membrane Strips in Microelectromechanical Systems (MEMS), J. Appl. Phys. 84 (1998), 2501.

[36] F. J. Tipler, Energy Conditions and Spacetime Singularities, Phys. Rev. D17 (1978), 2521.

[37] W. G. Unruh, Notes on Black Hole Evaporation, Phys. Rev. D14 (1976), 870.

[38] R. Verch, Local Definiteness, Primarity and Quasiequivalence of Quasifree Hadamard Quantum States in Curved Space-Time, Commun. Math. Phys. 160 (1994), 507.

[39] R. M. Wald, Trace Anomaly of a Conformally Invariant Quantum Field in Curved Space-Time, Phys. Rev. D17 (1978), 1477.

[40] R. M. Wald, General Relativity, University of Chicago Press, Chicago, IL (1984). 
[41] R. M. Wald, Quantum Field Theory in Curved Spacetime and Black Hole Thermodynamics, Chicago Lectures in Physics, University of Chicago Press, Chicago, IL (1994).

[42] R. M. Wald and U. Yurtsever, General Proof of the Averaged Null Energy Condition for a Massless Scalar Field in Two-dimensional Curved Space-Time, Phys. Rev. D44 (1991), 403.

Jan Schlemmer

Max-Planck-Inst. f. Mathematics in the Sciences

Inselstr. 22

D-04103 Leipzig

Germany

and

Institut f. Theoretische Physik

Universität Leipzig

Postfach 100920

D-04009 Leipzig

Germany

e-mail: schlemme@mis .mpg.de

Rainer Verch

Institut f. Theoretische Physik

Universität Leipzig

Postfach 100920

D-04009 Leipzig

Germany

e-mail: Rainer.Verch@itp.uni-leipzig.de

Communicated by Klaus Fredenhagen.

Submitted: February 27, 2008.

Accepted: May 5, 2008. 